\title{
2-(1,3-Dioxan-2-yl)ethylsulfonyl Group:
}

\section{A New Versatile Protecting and Activating Group for Amine Synthesis}

Izumi Sakamoto, Norimasa Izumi, Taeko Yamada, and Tetsuto Tsunoda*

Faculty of Pharmaceutical Sciences, Tokushima Bunri University, Tokushima 770-8514, Japan

\section{Supporting Information}

\section{Additional Information}

Table 1. Reaction of 8 using several Mitsunobu reagents

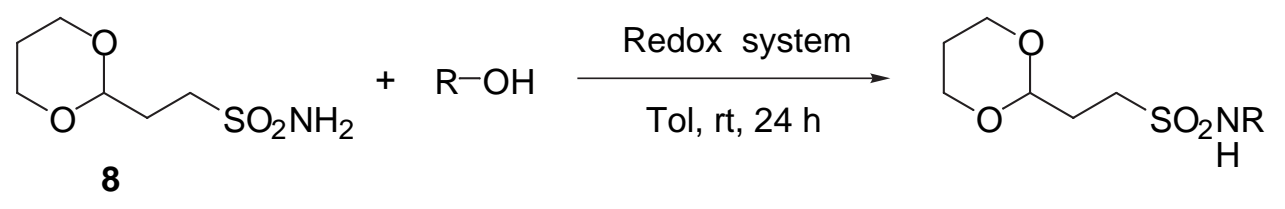

$\%$ yield of $N$-monoalkylated product

\begin{tabular}{lcccc}
\hline \multirow{2}{*}{$\mathrm{R}-\mathrm{OH}$} & \multicolumn{4}{c}{ Redox system } \\
\cline { 2 - 5 } & DEAD - TPP & TMAD - TBP & CMBP & CMMP \\
\hline $\mathrm{nyyyy}_{\mathrm{OH}}$ & trace & 42 & 67 & 6 \\
\hline
\end{tabular}

1) The reaction was carried out at $80^{\circ} \mathrm{C}$. 
Scheme 1. Alkylation reaction of $9 a$ with alkylbromide in the presence of base<smiles>CC(=O)OCCC1OCCCO1</smiles>

$9 a$

quant

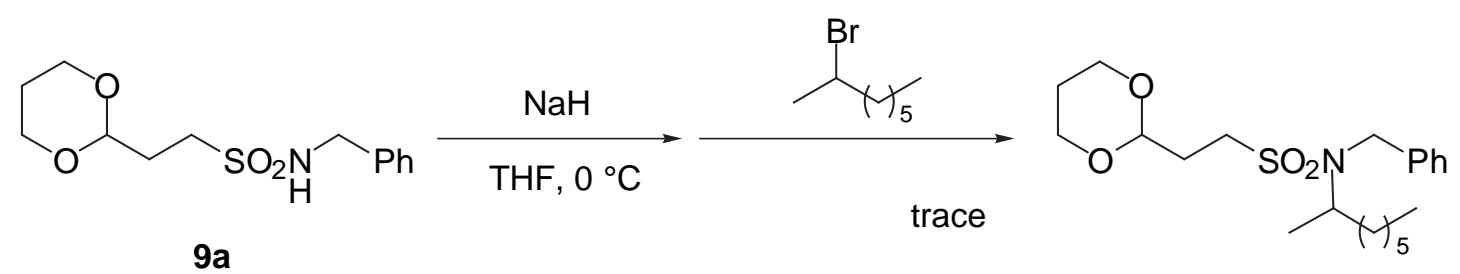

Table 2. Stability of Dios-amide

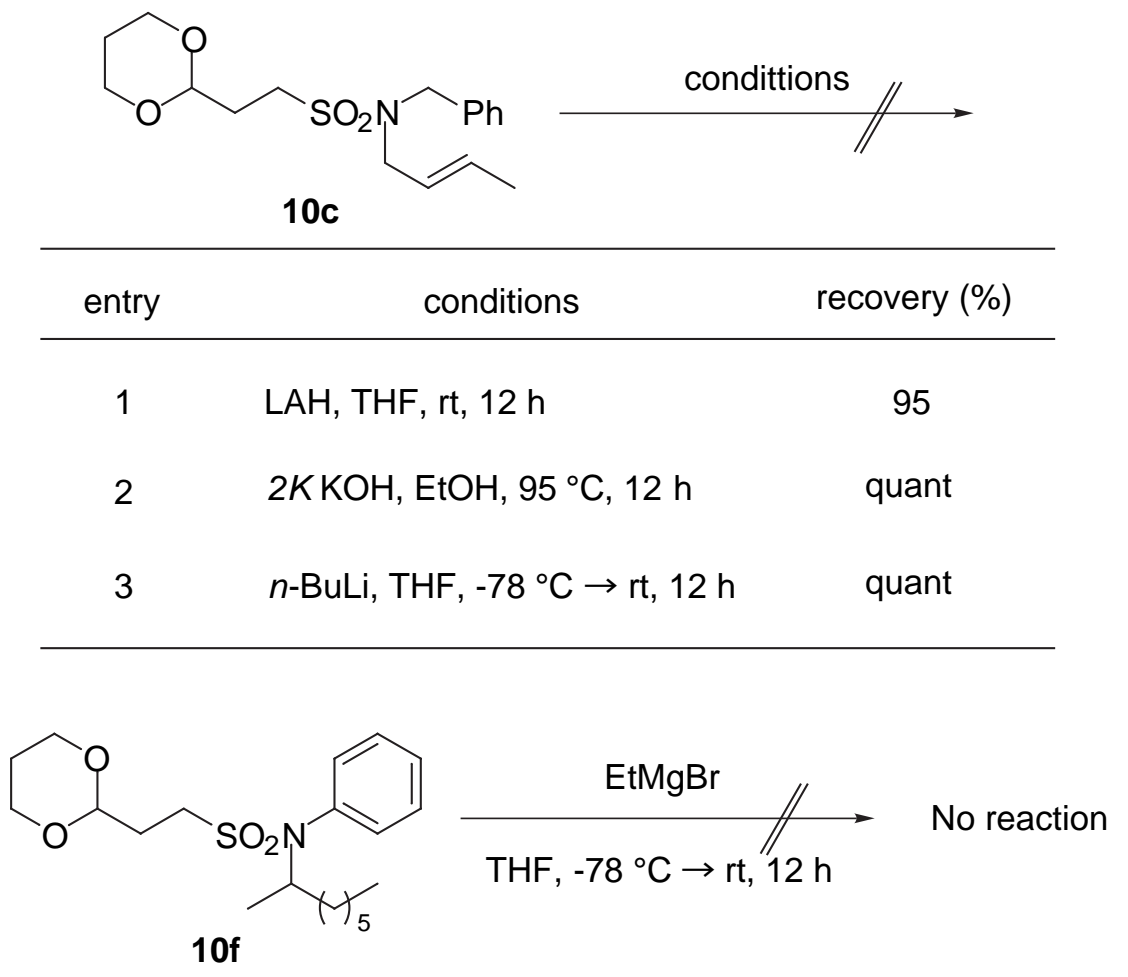




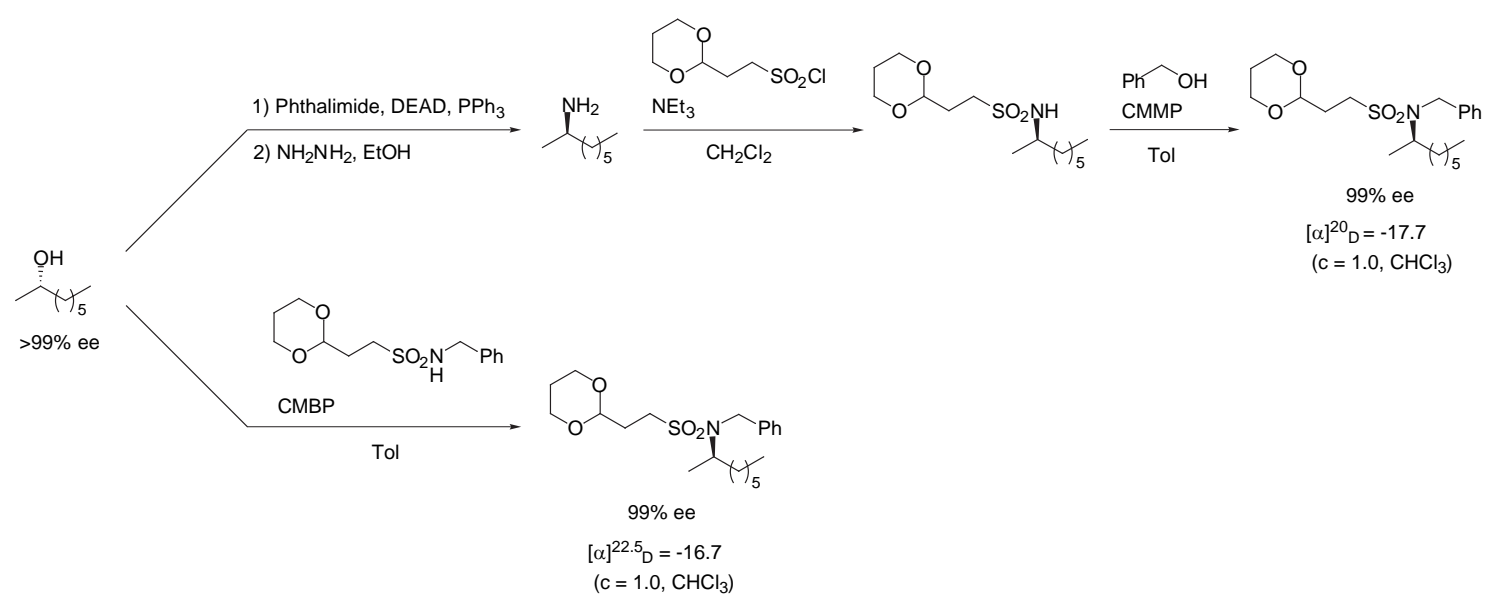

\section{Experimental procedures and compound characterization data}

\section{General}

Proton nuclear magnetic resonance $\left({ }^{1} \mathrm{H}\right.$ NMR) spectra and carbon nuclear magnetic resonance $\left({ }^{13} \mathrm{C}\right.$ NMR) were recorded on a Varian Mercury-300 (300 MHz) spectrometer. Chemical shifts for protons and carbons are reported in parts per million (ppm) downfield from tetramethylsilane as an internal standard. Data are presented as follows: chemical shift, multiplicity $(\mathrm{br}=$ broad, $\mathrm{s}=$ singlet, $\mathrm{d}=$ doublet, $\mathrm{t}=$ triplet, $\mathrm{q}=$ quartet, $\mathrm{m}=$ multiplet), coupling constant in Hertz (Hz) and integration. Infrared (IR) spectra were obtained using a JASCO FT/IR-410 spectrophotometer. Data are represented as frequency of absorption $\left(\mathrm{cm}^{-1}\right)$. Melting points were measured on a Büchi B-545 apparatus and are uncorrected. The mass spectroscopic data were obtained on a JEOL JMS-700 spectrophotometer. Optical rotations were measured on a JASCO DIP-1000 Digital Polarimeter at room temperature, using the sodium D line. Separations by column chromatography were performed on Fuji Silysia silica gel BW-127ZH. Reagents and solvents were commercial grades and were used as supplied with following exceptions: Toluene: distilled from calcium hydride and dried over calcium chloride. Alcohol and amine: distilled from calcium hydride. 


\section{Dios-Cl (7)}<smiles>ClCCC1OCCCO1</smiles>

6

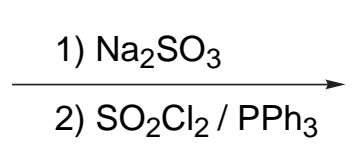

$83 \%$<smiles>O=S(=O)(Cl)CCC1OCCCO1</smiles>

7

A mixture of 2-(2-chloroethyl)-1,3-dioxane (6) $(6.4 \mathrm{~mL}, 50.6 \mathrm{mmol})$ and sodium sulfite $(7.6 \mathrm{~g}, 60.1 \mathrm{mmol})$ was refluxed in DME $(100 \mathrm{~mL})$ and water $(100 \mathrm{~mL})$ for 3 days. The resulting mixture was concentrated in vacuo, and then the residue was dried over $\mathrm{P}_{2} \mathrm{O}_{5}$ under reduced pressure $\left(100{ }^{\circ} \mathrm{C}, 5 \mathrm{~mm}\right)$ for $48 \mathrm{~h}$. This crude product, sodium 2-(1,3-dioxan-2-yl)ethylsulfonate, was suspended in $\mathrm{CH}_{2} \mathrm{Cl}_{2}(150 \mathrm{~mL})$, and then a solution of triphenylphosphine $(26.0 \mathrm{~g}, 99.2 \mathrm{mmol})$ and sulfuryl chloride $(9.0 \mathrm{~mL}$, $112.0 \mathrm{mmol})$ in $\mathrm{CH}_{2} \mathrm{Cl}_{2}(150 \mathrm{~mL})$ was added dropwise to the suspension at $0{ }^{\circ} \mathrm{C}$ under an argon atmosphere. After the resulting mixture was stirred at $0{ }^{\circ} \mathrm{C}$ for $2 \mathrm{~h}$, the reaction mixture was carefully poured into a mixture of water $(500 \mathrm{~mL})$ and hexane $(500 \mathrm{~mL})$ at $0{ }^{\circ} \mathrm{C}$. The organic layer was washed with water $(3 \mathrm{x} 500 \mathrm{~mL})$, dried $\left(\mathrm{MgSO}_{4}\right)$, filtered, and concentrated in vacuo. The residue was dissolved in hexane $(250 \mathrm{~mL})$, and then insoluble materials were filtered off. The filtrate was concentrated in vacuo, and the crude product was subjected to quick purification through a short silica gel column chromatography (eluent: ether / petroleum ether, 1/1, v/v) to yield Dios-chloride (7) as a colorless oil (9.02 g, $42.0 \mathrm{mmol}, 83 \%)$.

The title compound 7 was isolated as a colorless oil. ${ }^{1} \mathbf{H}$ NMR $\left(300 \mathbf{M H z}, \mathrm{CDCl}_{3}\right)$ $\delta 4.78(1 \mathrm{H}, \mathrm{t}, J=4.2 \mathrm{~Hz}), 4.10(2 \mathrm{H}, \mathrm{ddd}, J=11.9,4.8,1.2 \mathrm{~Hz}), 3.81-3.89(2 \mathrm{H}, \mathrm{m})$, $3.77(2 \mathrm{H}$, br t, $J=11.4 \mathrm{~Hz}), 2.30(2 \mathrm{H}, \mathrm{dt}, J=11.7,4.2 \mathrm{~Hz}), 1.95-2.13(1 \mathrm{H}, \mathrm{m}), 1.37(1 \mathrm{H}$, br d, $J=13.8 \mathrm{~Hz}) ;{ }^{13} \mathbf{C}$ NMR $\left(75 \mathrm{MHz}, \mathrm{CDCl}_{3}\right) \delta 98.0,66.7,60.1,29.7,25.3$; IR (neat, $\left.\mathrm{cm}^{-1}\right)$ 2974, 2930, 2859, 1145, 1128; MS (CI) m/z 215([M+H $]^{+}$, base peak), 115; HRMS calcd for $\mathrm{C}_{6} \mathrm{H}_{12} \mathrm{O}_{4} \mathrm{SCl} 215.0145\left([\mathrm{M}+\mathrm{H}]^{+}\right)$, found 215.0114 . 
2-(1,3-Dioxan-2-yl)ethylsulfonamide (8).

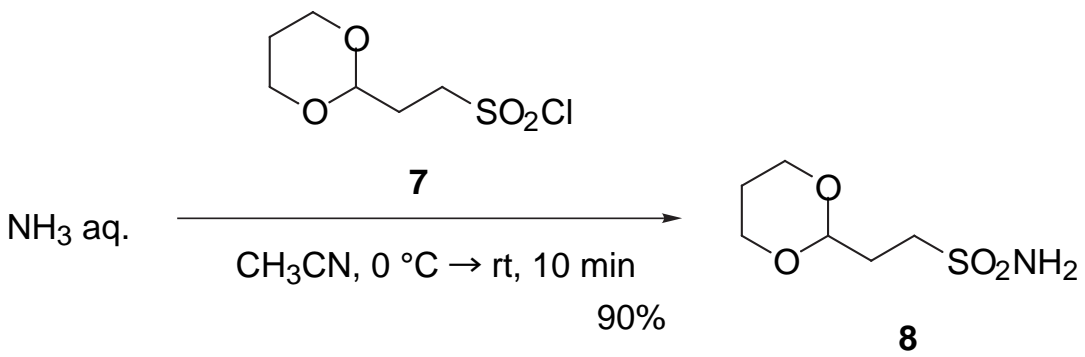

To a cooled solution of $7(1.0 \mathrm{~g}, 46.9 \mathrm{mmol})$ in acetonitrile $(5 \mathrm{~mL})$ was added dropwise aqueous ammonia $(15 \mathrm{~mL})$ over a period of $3 \mathrm{~min}$ at $0{ }^{\circ} \mathrm{C}$. The resulting mixture was stirred for $10 \mathrm{~min}$ at $\mathrm{rt}$, and concentrated in vacuo. The crude product was purified by silica gel column chromatography (eluent: hexane/AcOEt, 1/1 - 1/5, v/v) to yield $\mathbf{8}$ as a colorless solid (826 mg, $4.2 \mathrm{mmol}, 90 \%$ ).

The title compound 8 was isolated as colorless needle crystals; melting point: $74-75{ }^{\circ} \mathrm{C}$ (AcOEt-Hexane). ${ }^{1} \mathbf{H}$ NMR (300 MHz, $\left.\mathrm{CDCl}_{3}\right) \delta 5.18(2 \mathrm{H}$, br s), $4.73(1 \mathrm{H}, \mathrm{t}, J=4.8 \mathrm{~Hz}$ ), 4.10(2H, ddd, $J=11.9,5.0,1.2 \mathrm{~Hz}), 3.78(2 \mathrm{H}$, br t, $J=11.4 \mathrm{~Hz}), 3.23-3.31(2 \mathrm{H}, \mathrm{m})$, $2.14(2 \mathrm{H}, \mathrm{td}, J=7.5,4.8 \mathrm{~Hz}), 1.96-2.14(1 \mathrm{H}, \mathrm{m}), 1.36(1 \mathrm{H}, \mathrm{br} \mathrm{d}, J=13.8 \mathrm{~Hz}) ;{ }^{13} \mathbf{C} \mathbf{~ N M R}$ $\left(75 \mathrm{MHz}, \mathrm{CDCl}_{3}\right) \delta 99.4,66.8,49.8,29.5,25.4$; IR $\left(\mathrm{KBr}, \mathrm{cm}^{-1}\right)$ 3277, 2876, 1327, 1142; MS (EI) m/z 194([M-H] $]^{+}$), 87(base peak); HRMS calcd for $\mathrm{C}_{6} \mathrm{H}_{12} \mathrm{NO}_{4} \mathrm{~S} 194.0487$ ([M$\mathrm{H}]^{+}$), found 194.0465; Anal. calcd for $\mathrm{C}_{6} \mathrm{H}_{13} \mathrm{NO}_{4} \mathrm{~S}$ : C, 36.91; H, 6.71; N, 7.17. found: $\mathrm{C}$, 36.99; H, 6.86; N, 7.24.

\section{General procedure for sulfonylation of amine}

Reaction of 7 with benzylamine: $N$-Benzyl-2-(1,3-dioxan-2-yl)ethylsulfonamide(9a).

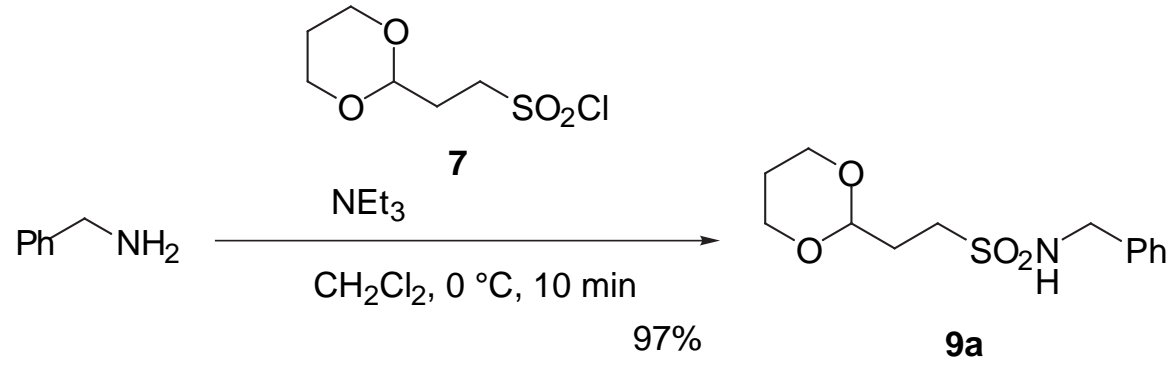


To a cooled solution $\left(0{ }^{\circ} \mathrm{C}\right)$ of benzylamine $(115 \mu \mathrm{L}, 1.05 \mathrm{mmol})$ and $\mathrm{Et}_{3} \mathrm{~N}(210 \mu \mathrm{L}, 1.5$ mmol $)$ in $\mathrm{CH}_{2} \mathrm{Cl}_{2}(3 \mathrm{~mL})$ was added $7(235 \mathrm{mg}, 1.1 \mathrm{mmol})$ with stirring. After addition, the mixture was stirred at $\mathrm{rt}$ for $10 \mathrm{~min}$, and then was quenched with a sat. $\mathrm{NH}_{4} \mathrm{Cl}$ aq. sol. (5 mL). The reaction mixture was extracted with $3 \times 5 \mathrm{~mL}$ portions of $\mathrm{CH}_{2} \mathrm{Cl}_{2}$, and the combined organic extracts were dried over $\mathrm{Na}_{2} \mathrm{SO}_{4}$, filtered, and concentrated under reduced pressure. The residue was subjected to purification by column chromatography on silica gel (eluent: hexane/AcOEt, 3/1 - 1/1, v/v) to give $291 \mathrm{mg}$ (97\%) of $N$-benzyl-2-(1,3-dioxan-2-yl)ethylsulfonamide (9a) as colorless crystals.

The title compound 9a was isolated as colorless needle crystals; melting point: $80-81{ }^{\circ} \mathrm{C}$ (AcOEt-Hexane). ${ }^{1} \mathbf{H}$ NMR (300 $\left.\mathrm{MHz}, \mathrm{CDCl}_{3}\right) \delta 7.26-7.40(5 \mathrm{H}, \mathrm{m}), 4.71(1 \mathrm{H}$, br s), $4.65(1 \mathrm{H}, \mathrm{t}, J=4.5 \mathrm{~Hz}), 4.29(2 \mathrm{H}, \mathrm{d}, J=5.7 \mathrm{~Hz}), 4.06(2 \mathrm{H}$, ddd, $J=11.9,4.8,1.2 \mathrm{~Hz})$, $3.74(2 \mathrm{H}$, br t, $J=11.2 \mathrm{~Hz}), 3.11-3.18(2 \mathrm{H}, \mathrm{m}), 2.07(2 \mathrm{H}, \mathrm{td}, J=7.5,4.5 \mathrm{~Hz}), 1.92-$ $2.08(1 \mathrm{H}, \mathrm{m}), 1.33(1 \mathrm{H}$, br d, $J=13.5 \mathrm{~Hz}) ;{ }^{13} \mathbf{C ~ N M R}\left(75 \mathrm{MHz}, \mathrm{CDCl}_{3}\right) \delta 136.9,128.7$, 127.8, 99.3, 66.7 47.3 47.1, 29.2, 25.4; IR (KBr, cm $\left.{ }^{-1}\right) 3274,2955,1128 ;$ MS (EI) m/z 284([M-H] $\left.]^{+}\right), 106$ (base peak); HRMS calcd for $\mathrm{C}_{13} \mathrm{H}_{18} \mathrm{NO}_{4} \mathrm{~S} 284.09565\left([\mathrm{M}-\mathrm{H}]^{+}\right)$, found 284.0952; Anal. calcd for $\mathrm{C}_{13} \mathrm{H}_{19} \mathrm{NO}_{4} \mathrm{~S}$ : C, 54.72; H, 6.71; N, 4.91. found: C, 54.86; $\mathrm{H}$, $6.82 ; \mathrm{N}, 4.89$.

\section{General procedure for Mitsunobu alkylation}

Reaction of $\mathbf{8}$ with 2-octen-1-ol: $\quad N$-(Octa-2-enyl)-2-(1,3-dioxan-2-yl)ethylsulfonamide(9b).

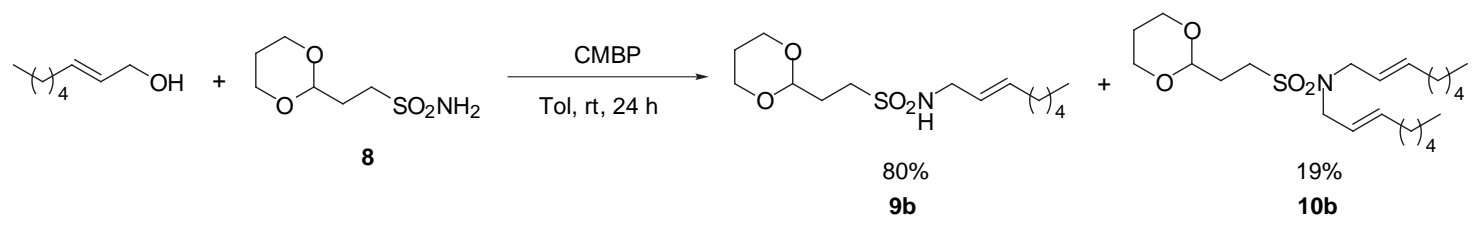

To a solution of 2-(1,3-dioxan-2-yl)ethylsulfonamide (8) $(295.3 \mathrm{mg}, 1.5 \mathrm{mmol})$ and 2octen-1-ol $(155 \mu \mathrm{L}, 1.0 \mathrm{mmol})$ in dry toluene $(5 \mathrm{~mL})$ was added CMBP $(0.4 \mathrm{~mL}, 1.5$ mmol) at $\mathrm{rt}$ under an argon atmosphere. The resulting mixture was stirred for $24 \mathrm{~h}$ at $\mathrm{rt}$ and concentrated in vacuo. The residue was purified by silica gel column chromatography (eluent: hexane/AcOEt, $5 / 1$ - 2/1, v/v) to yield $N$-(octen-2-yl)-2-(1,3dioxan-2-yl)ethylsulfonamide (9b) (242.9 mg, $0.80 \mathrm{mmol}, 80 \%)$ and , $N$-di(octa-2-enyl)- 
2-(1,3-dioxan-2-yl)ethylsulfonamide (10b) (40.4 mg, $0.097 \mathrm{mmol}, 14 \%)$.

The title compound 9b was isolated as a colorless oil. ${ }^{1} \mathbf{H}$ NMR (300 MHz, $\left.\mathrm{CDCl}_{3}\right) \delta 5.63-5.75(1 \mathrm{H}, \mathrm{m}), 5.41-5.52(1 \mathrm{H}, \mathrm{m}), 4.86(1 \mathrm{H}, \mathrm{t}, J=6.0 \mathrm{~Hz}), 4.69(1 \mathrm{H}, \mathrm{t}, J=$ $4.5 \mathrm{~Hz}), 4.08(2 \mathrm{H}, \mathrm{brd}, J=6.0 \mathrm{~Hz}), 3.76(2 \mathrm{H}$, br t,$J=12.0 \mathrm{~Hz}), 3.66(2 \mathrm{H}, \mathrm{td}, J=6.3,0.9$ $\mathrm{Hz}), 3.11-3.18(2 \mathrm{H}, \mathrm{m}), \quad 2.06(2 \mathrm{H}, \mathrm{td}, J=7.8,4.5 \mathrm{~Hz}), 1.94-2.11(3 \mathrm{H}, \mathrm{m}), 1.20-1.43(7 \mathrm{H}$, m), 0.89(3H, t, $J=6.6 \mathrm{~Hz}) ;{ }^{13} \mathbf{C}$ NMR $\left(75 \mathrm{MHz}, \mathrm{CDCl}_{3}\right) \delta 134.7,124.9,99.3,66.6,47.2$, 45.0, 31.9, 31.1, 29.1, 28.4, 25.3, 22.2, 13.8; IR (neat, $\mathrm{cm}^{-1}$ ) 3288, 2957, 2927, 2856, 1321, 1143; MS (EI) m/z 304([M-H] $\left.]^{+}\right), 126,87$ (base peak); HRMS calcd for $\mathrm{C}_{14} \mathrm{H}_{26} \mathrm{NO}_{4} \mathrm{~S} 304.15824\left([\mathrm{M}-\mathrm{H}]^{+}\right)$, found 304.1590.

The compound $\mathbf{1 0 b}$ was isolated as a colorless oil. ${ }^{1} \mathbf{H}$ NMR $\left(300 \mathrm{MHz}, \mathrm{CDCl}_{3}\right) \delta 5.32$ $5.45(2 \mathrm{H}, \mathrm{m}), 4.67(1 \mathrm{H}, \mathrm{t}, J=4.5 \mathrm{~Hz}), 4.08(2 \mathrm{H}, \mathrm{br} \mathrm{d}, J=6.0 \mathrm{~Hz}), 3.76(4 \mathrm{H}, \mathrm{d}, J=6.6 \mathrm{~Hz})$, $3.75(2 \mathrm{H}$, br t, $J=11.4 \mathrm{~Hz}), 3.04-3.12(2 \mathrm{H}, \mathrm{m}), 2.04(2 \mathrm{H}, \mathrm{td}, J=7.8,4.8 \mathrm{~Hz}), 1.95-$ 2.12(5H, m), 1.21-1.44(13H, m), 0.89(6H, t, $J=6.6 \mathrm{~Hz}) ;{ }^{13} \mathbf{C}$ NMR $(75 \mathrm{MHz}$, $\left.\mathrm{CDCl}_{3}\right) \delta 136.1,124.3,99.5,66.8,48.1,47.7,32.1,31.3,29.1,28.7,25.6,22.4,14.0$; IR (neat, $\mathrm{cm}^{-1}$ ) 2957, 2926, 2855, 1335, 1144; MS (EI) m/z 414([M-H $\left.]^{+}\right), 236$ (base peak), 114; HRMS calcd for $\mathrm{C}_{22} \mathrm{H}_{40} \mathrm{NO}_{4} \mathrm{~S} 414.26779$ ([M-H] $]^{+}$), found 414.2649.

Reaction of 9a with 2-buten-1-ol: $N$-Benzyl- $N$-crotyl-2-(1,3-dioxan-2-yl)ethylsulfonamide (10c).

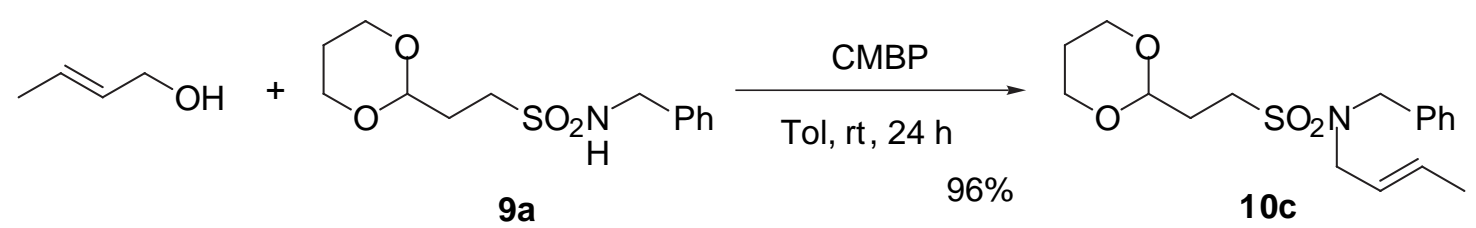

To a solution of $N$-benzyl-2-(1,3-dioxan-2-yl)ethylsulfonamide (9a) (430 mg, 1.5 $\mathrm{mmol})$ and 2-buten-1-ol $(85 \mu \mathrm{L}, 1.0 \mathrm{mmol})$ in dry toluene $(5 \mathrm{~mL})$ was added CMBP $(0.4 \mathrm{~mL}, 1.8 \mathrm{mmol})$ at $\mathrm{rt}$ under an argon atmosphere. The resulting mixture was stirred for $24 \mathrm{~h}$ at $\mathrm{rt}$ and concentrated in vacuo. The residue was purified by silica gel column chromatography (eluent: hexane/AcOEt, 10/1 - 5/1 - 2/1, v/v) to yield $N$-benzyl- $N$ crotyl-2-(1,3-dioxan-2-yl)ethylsulfonamide (10c) as a colorless solid (323 mg, 0.96 mmol, 96\%).

The title compound 10c was isolated as a colorless oil. ${ }^{1} \mathbf{H}$ NMR (300 MHz, 
$\left.\mathrm{CDCl}_{3}\right) \delta 7.25-7.38(5 \mathrm{H}, \mathrm{m}), 5.52-5.65(1 \mathrm{H}, \mathrm{m}), 5.37-5.49(1 \mathrm{H}, \mathrm{m}), 4.69(1 \mathrm{H}, \mathrm{t}, J=4.5 \mathrm{~Hz})$, $4.38(2 \mathrm{H}, \mathrm{s}), 4.09(2 \mathrm{H}, \mathrm{br} \mathrm{d}, J=5.4 \mathrm{~Hz}), 3.77(2 \mathrm{H}, \mathrm{br} \mathrm{t}, J=12.0 \mathrm{~Hz}), 3.69(2 \mathrm{H}, \mathrm{d}, J=6.6$ $\mathrm{Hz}), 3.09-3.17(2 \mathrm{H}, \mathrm{m}), 2.09(2 \mathrm{H}, \mathrm{dt}, J=10.5,5.1 \mathrm{~Hz}), 1.97-2.14(1 \mathrm{H}, \mathrm{m}), 1.70(3 \mathrm{H}, \mathrm{br} \mathrm{d}$, $J=6.2 \mathrm{~Hz}), 1.35(1 \mathrm{H}$, br d, $J=13.5 \mathrm{~Hz}) ;{ }^{13} \mathrm{C}$ NMR $\left(75 \mathrm{MHz}, \mathrm{CDCl}_{3}\right) \delta 136.1,131.2$, 128.3, 128.2 , 128.1, 127.5, 124.8, 99.3, 66.6, 49.6, 48.1, 47.6, 29.0, 25.4, 17.5; IR (neat, $\left.\mathrm{cm}^{-1}\right)$ 2965, 2856, 1330, 1143; MS (EI) m/z 338([M-H] $]^{+}$), 160(base peak), 114, 91; HRMS calcd for $\mathrm{C}_{17} \mathrm{H}_{24} \mathrm{NO}_{4} \mathrm{~S} 338.14259\left([\mathrm{M}-\mathrm{H}]^{+}\right)$, found 338.1422.

\section{Spectra Data}

$N$-Cetyl-2-(1,3-dioxan-2-yl)ethylsulfonamide (9c).<smiles>CC(C)CNS(=O)(=O)CCC1OCCCO1</smiles>

9c

The title compound 9c was isolated as colorless needle crystals; melting point: $86-87^{\circ} \mathrm{C}$ (AcOEt-Hexane). ${ }^{1} \mathbf{H}$ NMR $\left(300 \mathrm{MHz}, \mathrm{CDCl}_{3}\right) \delta 4.70(1 \mathrm{H}, \mathrm{t}, J=4.8 \mathrm{~Hz}), 4.21(1 \mathrm{H}, \mathrm{t}, J=$ $6.3 \mathrm{~Hz}), 4.09(2 \mathrm{H}$, br d, $J=6.0 \mathrm{~Hz}), 3.77(2 \mathrm{H}$, br t, $J=12.0 \mathrm{~Hz}), 3.13-3.20(2 \mathrm{H}, \mathrm{m}), 3.13-$ $3.05(2 \mathrm{H}, \mathrm{m}), 2.07(2 \mathrm{H}, \mathrm{td}, J=7.5,4.5 \mathrm{~Hz}), 1.96-2.13(1 \mathrm{H}, \mathrm{m}), 1.48-1.62(2 \mathrm{H}, \mathrm{m}), 1.24-$ $1.40(27 \mathrm{H}, \mathrm{m}), 0.88(3 \mathrm{H}, \mathrm{t}, J=6.6 \mathrm{~Hz}) ;{ }^{13} \mathrm{C}$ NMR $\left(75 \mathrm{MHz}, \mathrm{CDCl}_{3}\right) \delta 99.4,66.8,46.8$, 43.3, 31.9, 30.3, 29.7, 29.5, 29.5, 29.4, 29.3, 29.1, 26.6, 25.6, 22.7, 14.1; IR $\left(\mathrm{KBr}, \mathrm{cm}^{-1}\right)$ 2851, 1312, 1136; MS (EI) m/z 418([M-H] $\left.]^{+}\right), 344,240,179,114$ (base peak); HRMS calcd for $\mathrm{C}_{22} \mathrm{H}_{44} \mathrm{NO}_{4} \mathrm{~S} 418.29908\left([\mathrm{M}-\mathrm{H}]^{+}\right)$, found 418.2953; Anal. calcd for $\mathrm{C}_{22} \mathrm{H}_{45} \mathrm{NO}_{4} \mathrm{~S}: \mathrm{C}, 62.97 ; \mathrm{H}, 10.81 ; \mathrm{N}, 3.34$. found: $\mathrm{C}, 63.10 ; \mathrm{H}, 11.19 ; \mathrm{N}, 3.20$.

$N$-(1-Methylheptanyl)-2-(1,3-dioxan-2-yl)ethylsulfonamide (9d).<smiles>CC(C)NC(C)S(=O)(=O)OCCC1OCCCO1</smiles> 
The title compound 9d was isolated as a colorless oil. ${ }^{1} \mathbf{H}$ NMR (300 MHz, $\left.\mathrm{CDCl}_{3}\right) \delta 4.70(1 \mathrm{H}, \mathrm{t}, J=4.8 \mathrm{~Hz}), 4.09(3 \mathrm{H}$, br d, $J=5.4 \mathrm{~Hz}), 3.76(2 \mathrm{H}$, br t,$J=11.4 \mathrm{~Hz})$, $3.45(1 \mathrm{H}$, sextet, $J=6.3 \mathrm{~Hz}) 3.11-3.18(2 \mathrm{H}, \mathrm{m}), 2.08(2 \mathrm{H}, \mathrm{td}, J=7.8,4.8 \mathrm{~Hz}), 1.96-$ $2.13(1 \mathrm{H}, \mathrm{m}), 1.35(1 \mathrm{H}$, br d, $J=13.5 \mathrm{~Hz}), 1.24-1.52(10 \mathrm{H}, \mathrm{m}), 1.22(3 \mathrm{H}, \mathrm{d}, J=6.3 \mathrm{~Hz})$, $0.88(3 \mathrm{H}, \mathrm{t}, J=6.9 \mathrm{~Hz}) ;{ }^{13} \mathrm{C} \mathbf{N M R}\left(75 \mathrm{MHz}, \mathrm{CDCl}_{3}\right) \delta 99.3,66.6,50.0,48.1,37.7,31.6$, 29.3, 28.9, 25.6, 25.4, 22.4, 22.1, 13.9 ; IR (neat, $\mathrm{cm}^{-1}$ ) 3297, 2930, 1132; MS (EI) m/z 306([M-H] $]^{+}$), 222, 179(base peak), 103; HRMS calcd for $\mathrm{C}_{14} \mathrm{H}_{28} \mathrm{NO}_{4} \mathrm{~S} 306.17389$ $\left([\mathrm{M}-\mathrm{H}]^{+}\right)$, found 306.1751 .

$N$-Phenyl-2-(1,3-dioxan-2-yl)ethylsulfonamide (9e).<smiles>O=S(=O)(O)Nc1ccccc1</smiles>

$9 e$

The title compound 9e was isolated as colorless needle crystals; melting point: 110$112{ }^{\circ} \mathrm{C}$ (AcOEt-Hexane). ${ }^{1} \mathbf{H}$ NMR $\left(300 \mathrm{MHz}, \mathrm{CDCl}_{3}\right) \delta 7.14-7.47(5 \mathrm{H}, \mathrm{m}), 6.45(1 \mathrm{H}, \mathrm{br}$ s), $4.68(1 \mathrm{H}, \mathrm{t}, J=4.8 \mathrm{~Hz}), 4.06(2 \mathrm{H}$, br d, $J=10.8 \mathrm{~Hz}), 3.73(2 \mathrm{H}$, br t, $J=12.0 \mathrm{~Hz})$, $3.22-3.30(2 \mathrm{H}, \mathrm{m}), 2.11(2 \mathrm{H}, \mathrm{td}, J=7.8,4.8 \mathrm{~Hz}), 1.92-2.22(1 \mathrm{H}, \mathrm{m}), 1.32(1 \mathrm{H}, \mathrm{br} \mathrm{d}, J=$ $13.5 \mathrm{~Hz}) ;{ }^{13} \mathbf{C}$ NMR $\left(75 \mathrm{MHz}, \mathrm{CDCl}_{3}\right) \delta 136.7,129.5,125.0,120.5,99.2,99.2,66.7$, 46.5, 29.1, 25.4; IR (KBr, cm $\left.{ }^{-1}\right)$ 3259, 2969, 2860, 1338; MS (EI) m/z 271( $\left.\mathrm{M}^{+}\right), 115$, 93(base peak); HRMS calcd for $\mathrm{C}_{12} \mathrm{H}_{17} \mathrm{NO}_{4} \mathrm{~S} 271.08782\left(\mathrm{M}^{+}\right)$, found 271.0868; Anal. calcd for $\mathrm{C}_{12} \mathrm{H}_{17} \mathrm{NO}_{4} \mathrm{~S}$ : C, 53.12; H, 6.32; N, 5.16. found: C, 52.74; H, 5.93; N, 4.98.

$N^{\prime}$-(tert-Butoxycarbonyl)- $N$-[2-(1,3-dioxan-2-yl)ethylsulfonyl]-1,3-propanediamine (9f).

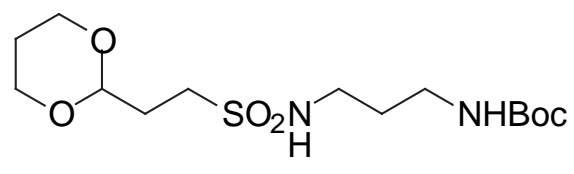


The title compound 9 f was isolated as colorless needle crystals; melting point: $75-77{ }^{\circ} \mathrm{C}$ (AcOEt-Hexane). ${ }^{1} \mathbf{H}$ NMR (300 MHz, $\left.\mathrm{CDCl}_{3}\right) \delta 5.19(1 \mathrm{H}$, br s), 4.79(1H, br s), 4.70(1H, t, $J=4.2 \mathrm{~Hz}), 4.09(2 \mathrm{H}$, br d, $J=5.4 \mathrm{~Hz}), 3.76(2 \mathrm{H}, \mathrm{br} \mathrm{t}, J=12.2 \mathrm{~Hz}), 3.24(2 \mathrm{H}, \mathrm{br} \mathrm{q}, J=$ $5.7 \mathrm{~Hz}), 3.10-3.20(4 \mathrm{H}, \mathrm{m}), 2.08(2 \mathrm{H}, \mathrm{dt}, J=11.4,4.5 \mathrm{~Hz}), 1.95-2.14(1 \mathrm{H}, \mathrm{m}), 1.69(2 \mathrm{H}$, quintet, $J=6.3 \mathrm{~Hz}), 1.44(9 \mathrm{H}, \mathrm{s}), 1.35(1 \mathrm{H}, \mathrm{br} \mathrm{d}, J=13.5 \mathrm{~Hz}) ;{ }^{13} \mathbf{C} \mathbf{N M R}(75 \mathrm{MHz}$, $\left.\mathrm{CDCl}_{3}\right) \delta 156.6,99.3,79.4,66.7,46.8,39.9,36.8,30.7,29.3,28.3,25.5$; IR $\left(\mathrm{KBr}, \mathrm{cm}^{-1}\right)$ 3558, 3370, 2973, 2935, 2377, 1690, 1319, 1143; MS (CI) m/z 353([M+H] $\left.]^{+}\right), 297,253$, 221(base peak); HRMS calcd for $\mathrm{C}_{14} \mathrm{H}_{29} \mathrm{~N}_{2} \mathrm{O}_{6} \mathrm{~S} 353.17462\left([\mathrm{M}+\mathrm{H}]^{+}\right)$, found 353.1737; Anal. calcd for $\mathrm{C}_{14} \mathrm{H}_{28} \mathrm{~N}_{2} \mathrm{O}_{6} \mathrm{~S}: \mathrm{C}, 47.71 ; \mathrm{H}, 8.01 ; \mathrm{N}, 7.95$. found: C, 47.87; H, 8.13; N, 7.80 .

$N, N$-Dibenzyl-2-(1,3-dioxan-2-yl)ethylsulfonamide (10a).<smiles>O=S(CCC1OCCCO1)N(Cc1ccccc1)c1ccccc1</smiles>

10a

The title compound 10a was isolated as colorless needle crystals; melting point: 67$69{ }^{\circ} \mathrm{C}$ (AcOEt-Hexane). ${ }^{1} \mathrm{H}$ NMR (300 MHz, $\left.\mathrm{CDCl}_{3}\right) \delta 7.24-7.37(10 \mathrm{H}, \mathrm{m}), 4.66(1 \mathrm{H}, \mathrm{t}, J$ $=4.8 \mathrm{~Hz}), 4.33(4 \mathrm{H}, \mathrm{s}), 4.08(2 \mathrm{H}, \mathrm{br} \mathrm{d}, J=5.4 \mathrm{~Hz}), 3.75(2 \mathrm{H}, \mathrm{br} \mathrm{t}, J=12.0 \mathrm{~Hz}), 3.08-$ $3.15(2 \mathrm{H}, \mathrm{m}), 2.08(2 \mathrm{H}, \mathrm{td}, J=8.1,5.1 \mathrm{~Hz}), 1.95-2.12(1 \mathrm{H}, \mathrm{m}), 1.34(1 \mathrm{H}, \mathrm{br} \mathrm{d}, J=13.5$ $\mathrm{Hz}) ;{ }^{13} \mathrm{C}$ NMR $\left(75 \mathrm{MHz}, \mathrm{CDCl}_{3}\right) \delta 135.6,128.6,128.6,127.8,99.4,66.8,50.0,48.1$, 29.1, 25.6; IR $\left(\mathrm{KBr}, \mathrm{cm}^{-1}\right)$ 2968, 2856, 1331, 1143; MS (EI) m/z 374([M-H] $), 196$, 91(base peak); HRMS calcd for $\mathrm{C}_{20} \mathrm{H}_{24} \mathrm{NO}_{4} \mathrm{~S} 374.14259$ ([M-H] $]^{+}$), found 374.1438; Anal. calcd for $\mathrm{C}_{20} \mathrm{H}_{25} \mathrm{NO}_{4} \mathrm{~S}$ : C, 63.98; H, 6.71; N, 3.73. found: C, 63.90; H, 6.76; N, 3.52 .

$N$-Benzyl- $N$-cetyl-2-(1,3-dioxan-2-yl)ethylsulfonamide (10d).<smiles>[Y14]CN(Cc1ccccc1)OCCC1OCCCO1</smiles> 
The title compound 10d was isolated as colorless needle crystals; melting point: 51$53{ }^{\circ} \mathrm{C}$ (AcOEt-Hexane). ${ }^{1} \mathbf{H}$ NMR (300 MHz, $\left.\mathrm{CDCl}_{3}\right) \delta 7.25-7.38(5 \mathrm{H}, \mathrm{m}), 4.69(1 \mathrm{H}, \mathrm{t}, J$ $=4.5 \mathrm{~Hz}), 4.40(2 \mathrm{H}, \mathrm{s}), 4.09(2 \mathrm{H}$, br d, $J=5.7 \mathrm{~Hz}), 3.76(2 \mathrm{H}$, br t,$J=12.0 \mathrm{~Hz}), 3.07-$ $3.17(4 \mathrm{H}, \mathrm{m}), 1.96-2.14(3 \mathrm{H}, \mathrm{m}), 1.13-1.53(29 \mathrm{H}, \mathrm{m}), 0.88(3 \mathrm{H}, \mathrm{t}, J=6.3 \mathrm{~Hz}) ;{ }^{13} \mathbf{C}$ NMR $\left(75 \mathrm{MHz}, \mathrm{CDCl}_{3}\right) \delta 136.6,128.6,128.3,127.7,99.5,66.8,51.1,47.4,47.0,31.9,29.6$, 29.6, 29.5, 29.4, 29.3, 29.1, 29.1, 28.1, 26.6, 25.6, 22.6, 14.1; IR $\left(\mathrm{KBr}, \mathrm{cm}^{-1}\right) 2924$, 2853, 1335, 1144; MS (EI) m/z 508([M-H] $\left.]^{+}\right), 330,179,114$ (base peak), 91; HRMS calcd for $\mathrm{C}_{29} \mathrm{H}_{50} \mathrm{NO}_{4} \mathrm{~S} 508.34603\left([\mathrm{M}-\mathrm{H}]^{+}\right)$, found 508.3469; Anal. calcd for $\mathrm{C}_{29} \mathrm{H}_{51} \mathrm{NO}_{4} \mathrm{~S}: \mathrm{C}, 68.33 ; \mathrm{H}, 10.08 ; \mathrm{N}, 2.75$. found: $\mathrm{C}, 68.17 ; \mathrm{H}, 10.36 ; \mathrm{N}, 2.86$.

$N$-Benzyl- $N$-(1-methylheptanyl)-2-(1,3-dioxan-2-yl)ethylsulfonamide (10e).

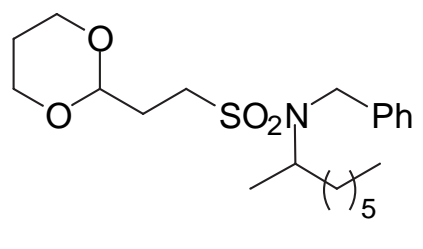

$10 e$

The title compound 10e was isolated as a colorless oil. ${ }^{1} \mathbf{H}$ NMR (300 MHz, $\left.\mathrm{CDCl}_{3}\right) \delta 7.20-7.44(5 \mathrm{H}, \mathrm{m}), 4.63(1 \mathrm{H}, \mathrm{t}, J=4.8 \mathrm{~Hz}), 4.39(1 \mathrm{H}, \mathrm{d}, J=15.6 \mathrm{~Hz}), 4.29(1 \mathrm{H}, \mathrm{d}$, $J=15.6 \mathrm{~Hz}), 4.05(2 \mathrm{H}$, br d, $J=5.4 \mathrm{~Hz}), 3.83(1 \mathrm{H}$, sextet, $J=6.9 \mathrm{~Hz}), 3.73(2 \mathrm{H}, \mathrm{br} \mathrm{t}, J=$ $11.9 \mathrm{~Hz}), 2.90-3.07(2 \mathrm{H}, \mathrm{m}), 1.88-2.10(3 \mathrm{H}, \mathrm{m}), 1.16(3 \mathrm{H}, \mathrm{d}, J=6.9 \mathrm{~Hz}), 1.04-1.48(26 \mathrm{H}$, $\mathrm{m}), 0.85(3 \mathrm{H}, \mathrm{t}, J=6.6 \mathrm{~Hz}) ;{ }^{13} \mathbf{C}$ NMR $\left(75 \mathrm{MHz}, \mathrm{CDCl}_{3}\right) \delta 138.3,128.2,128.2,127.3$, 99.4, 66.6, 54.4, 47.6, 46.9, 35.6, 31.5, 29.0, 28.8, 26.6, 25.4, 22.4, 19.6, 13.9; IR (neat, $\left.\mathrm{cm}^{-1}\right)$ 2929, 2856, 1332, 1140; MS (EI) m/z 396([M-H] $\left.]^{+}\right), 312$, 134, 91(base peak); HRMS calcd for $\mathrm{C}_{21} \mathrm{H}_{34} \mathrm{NO}_{4} \mathrm{~S} 396.22084\left([\mathrm{M}-\mathrm{H}]^{+}\right)$, found 396.2195.

\section{$N$-(1-Methylheptanyl)- $N$-phenyl-2-(1,3-dioxan-2-yl)ethylsulfonamide (10f ).}<smiles>CC(C)C(C)Nc1ccccc1N(C)C(C)C</smiles>

$10 f$ 
The title compound 10f was isolated as a colorless oil. ${ }^{1} \mathbf{H}$ NMR (300 MHz, $\left.\mathrm{CDCl}_{3}\right) \delta 7.24-7.43(5 \mathrm{H}, \mathrm{m}), 4.67(1 \mathrm{H}, \mathrm{t}, J=4.5 \mathrm{~Hz}), 4.25(1 \mathrm{H}$, sextet, $J=6.9 \mathrm{~Hz})$, $4.06(2 \mathrm{H}$, br d, $J=5.4 \mathrm{~Hz}), 3.73(2 \mathrm{H}$, br t,$J=12.9 \mathrm{~Hz}), 3.10-3.20(2 \mathrm{H}, \mathrm{m}), 2.10(2 \mathrm{H}, \mathrm{dt}, J$ $=10.8,4.8 \mathrm{~Hz}), 1.92-2.11(1 \mathrm{H}, \mathrm{m}), 1.15-1.57(26 \mathrm{H}, \mathrm{m}), 1.17(3 \mathrm{H}, \mathrm{d}, J=6.9 \mathrm{~Hz}), 0.88(3 \mathrm{H}$, $\mathrm{t}, J=6.6 \mathrm{~Hz}) ;{ }^{13} \mathrm{C}$ NMR $\left(75 \mathrm{MHz}, \mathrm{CDCl}_{3}\right) \delta 135.1,132.2,128.8,128.4,99.3,66.6,55.4$, 47.1, 35.7, 31.5, 29.2, 28.9, 26.5, 25.4, 22.4, 20.6, 13.9; IR (neat, $\mathrm{cm}^{-1}$ ) 2956, 2930, 2856, 1337, 1146; MS (EI) m/z 383(M+), 298, 179, 120(base peak); HRMS calcd for $\mathrm{C}_{20} \mathrm{H}_{33} \mathrm{NO}_{4} \mathrm{~S} 383.21301\left(\mathrm{M}^{+}\right)$, found 383.2124.

$N$-benzyl- $N^{\prime}$-(tert-Butoxycarbonyl)- $N$-[2-(1,3-dioxan-2-yl)ethylsulfonyl]-1,3propanediamine $(\mathbf{1 0 g})$.

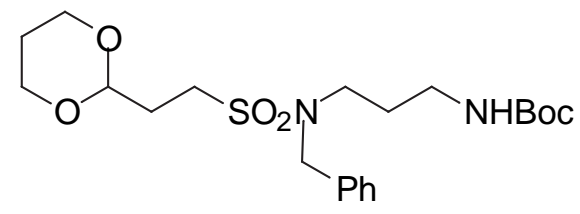

$10 \mathrm{~g}$

The title compound $\mathbf{1 0 g}$ was isolated as colorless needle crystals; melting point: 100$103{ }^{\circ} \mathrm{C}$ (AcOEt-Hexane). ${ }^{1} \mathbf{H}$ NMR $\left(300 \mathrm{MHz}, \mathrm{CDCl}_{3}\right) \delta 7.27-7.38(5 \mathrm{H}, \mathrm{m}), 4.85(1 \mathrm{H}$, br s), $4.68(1 \mathrm{H}, \mathrm{t}, J=4.8 \mathrm{~Hz}), 4.38(1 \mathrm{H}, \mathrm{s}), 4.09(2 \mathrm{H}$, br d, $J=5.4 \mathrm{~Hz}), 3.76(2 \mathrm{H}$, br t, $J=$ $11.6 \mathrm{~Hz}), 3.23(2 \mathrm{H}, \mathrm{t}, J=6.9 \mathrm{~Hz}), 3.07-3.16(2 \mathrm{H}, \mathrm{m}), 3.04(2 \mathrm{H}, \mathrm{br} \mathrm{q}, J=5.7 \mathrm{~Hz}), 2.08(2 \mathrm{H}$, $\mathrm{dt}, J=11.4,4.5 \mathrm{~Hz}), 1.95-2.14(1 \mathrm{H}, \mathrm{m}), 1.54(2 \mathrm{H}$, quintet, $J=6.3 \mathrm{~Hz}), 1.42(9 \mathrm{H}, \mathrm{s})$, $1.35(1 \mathrm{H}$, br d, $J=13.5 \mathrm{~Hz}) ;{ }^{13} \mathbf{C}$ NMR $\left(75 \mathrm{MHz}, \mathrm{CDCl}_{3}\right) \delta 155.9,136.2,128.7,128.4$, 128.0, 99.3, 79.0, 66.8, 51.8, 46.4, 45.1, 36.9, 29.0, 28.3, 25.5; IR (KBr, $\left.\mathrm{cm}^{-1}\right) 3383$, 2973, 2932, 2858, 2360, 1708, 1365, 1144; MS (EI) m/z 443([M+H] $\left.]^{+}\right), 387,343$ (base peak), 263, 207; HRMS calcd for $\mathrm{C}_{21} \mathrm{H}_{35} \mathrm{~N}_{2} \mathrm{O}_{6} \mathrm{~S} 443.22156\left([\mathrm{M}+\mathrm{H}]^{+}\right)$, found 443.2222; Anal. calcd for $\mathrm{C}_{21} \mathrm{H}_{34} \mathrm{~N}_{2} \mathrm{O}_{6} \mathrm{~S}$ : C, 56.99; H, 7.74; N, 6.33. found: C, 57.21; H, 7.86; N, 6.19 . 
General procedure for Desulfurization

Procedure 1: Reaction of 10c<smiles>C/C=C\CN(Cc1ccccc1)S(=O)(=O)[O-]</smiles><smiles>C/C=C\CNCc1ccccc1</smiles>

A solution of $N$-benzyl- $N$-crotyl-2-(1,3-dioxan-2-yl)ethylsulfonamide (10c) $(340 \mathrm{mg}$, $1.00 \mathrm{mmol})$ in $80 \%$ aqueous trifluoroacetic acid $(5 \mathrm{~mL})$ was heated at $65{ }^{\circ} \mathrm{C}$ for $90 \mathrm{~min}$. After cooling to $\mathrm{rt}$, the reaction mixture was diluted with ether $(20 \mathrm{~mL})$ and extracted with $2 \mathrm{M} \mathrm{HCl}$ aq. ( $3 \times 20 \mathrm{~mL}$ ). The combined aqueous layers were treated with sat. $\mathrm{NaHCO}_{3}$ aq. $(100 \mathrm{~mL})$ at $0{ }^{\circ} \mathrm{C}$, and the mixture was extracted with $\mathrm{CH}_{2} \mathrm{Cl}_{2}(3 \times 100 \mathrm{~mL})$. The organic layers were dried $\left(\mathrm{K}_{2} \mathrm{CO}_{3}\right)$, filtered, concentrated in vacuo to afford pure $N$ crotylbenzylamine as a pale yellow oil (151 mg, $0.93 \mathrm{mmol}, 93 \%)$.

Procedure 2: Reaction of 10e<smiles>CC(NCc1ccccc1)C(C)NC(=O)OCCC1OCCCO1</smiles>

A solution of $N$-benzyl- $N$-(1-methylheptanyl)-2-(1,3-dioxan-2-yl)ethylsulfonamide (10e) $(244.8 \mathrm{mg}, 0.62 \mathrm{mmol})$ in $80 \%$ aqueous trifluoroacetic acid $(3 \mathrm{~mL})$ was heated at $65{ }^{\circ} \mathrm{C}$ for $80 \mathrm{~min}$. After cooling to $0{ }^{\circ} \mathrm{C}$, the reaction mixture was treated with sat. $\mathrm{NaHCO}_{3}(60 \mathrm{~mL})$ and extracted with $\mathrm{CH}_{2} \mathrm{Cl}_{2}(3 \times 60 \mathrm{~mL})$. The organic portion was dried $\left(\mathrm{K}_{2} \mathrm{CO}_{3}\right)$, filtered, and concentrated in vacuo. The residue was subjected to purification by column chromatography on silica gel (eluent: hexane/AcOEt/Et ${ }_{3} \mathrm{~N}$, $50 / 10 / 0.6, \mathrm{v} / \mathrm{v})$ to give $N$-benzyl-1-methyloctylamine as a pale yellow oil (133.7 $\mathrm{mg}$, $0.61 \mathrm{mmol}, 99 \%)$. 
Selective deprotection of Boc group

Reaction of $\mathbf{1 0 g}$<smiles>CC(F)(OC(=O)OC(=O)NCCCN(Cc1ccccc1)S(=O)(=O)OCCC1OCCCO1)C(F)(F)F</smiles><smiles>NCCCN(Cc1ccccc1)S(=O)(=O)CCC1OCCCO1</smiles>

A solution of $N$-benzyl- $N$ '-(tert-butoxycarbonyl)- $N$-[2-(1,3-dioxan-2-yl)ethylsulfonyl]1,3-propanediamine $(\mathbf{1 0 g})(133.6 \mathrm{mg}, 0.30 \mathrm{mmol})$ in $80 \%$ aqueous trifluoroacetic acid $(1.5 \mathrm{~mL})$ was stirred at $0{ }^{\circ} \mathrm{C}$ for $1 \mathrm{hr}$. The reaction mixture was treated with sat. $\mathrm{NaHCO}_{3}(50 \mathrm{~mL})$ and extracted with $\mathrm{CH}_{2} \mathrm{Cl}_{2}(3 \mathrm{x} 50 \mathrm{~mL})$. The organic portion was dried $\left(\mathrm{K}_{2} \mathrm{CO}_{3}\right)$, filtered, and concentrated in vacuo. The residue was subjected to purification by column chromatography on silica gel (eluent: hexane $/ \mathrm{Et}_{3} \mathrm{~N}, 10 / 0.1$, v/v) to give $N$-benzyl- $N$-[2-(1,3-dioxan-2-yl)ethylsulfonyl]-1,3-propanediamine (11) as a colorless oil (92.1 mg, $0.27 \mathrm{mmol}, 90 \%)$.

The title compound $\mathbf{1 1}$ was isolated as a colorless oil. ${ }^{1} \mathbf{H}$ NMR (300 MHz, $\left.\mathrm{CDCl}_{3}\right) \delta 7.26-7.40(5 \mathrm{H}, \mathrm{m}), 4.68(1 \mathrm{H}, \mathrm{t}, J=4.5 \mathrm{~Hz}), 4.40(2 \mathrm{H}, \mathrm{s}), 4.08(2 \mathrm{H}, \mathrm{br} \mathrm{d}, J=6.0$ $\mathrm{Hz}), 3.76(2 \mathrm{H}$, br t, $J=11.4 \mathrm{~Hz}), 3.25(2 \mathrm{H}, \mathrm{t}, J=7.2 \mathrm{~Hz}), 3.08-3.16(2 \mathrm{H}, \mathrm{m}), 2.62(2 \mathrm{H}, \mathrm{t}, J$ $=6.3 \mathrm{~Hz}), 2.08(2 \mathrm{H}, \mathrm{dt}, J=10.8,4.8 \mathrm{~Hz}), 1.95-2.13(1 \mathrm{H}, \mathrm{m}), 1.57(2 \mathrm{H}$, quintet, $J=6.9$ $\mathrm{Hz}), 1.34(1 \mathrm{H}$, br d, $J=13.5 \mathrm{~Hz}) ;{ }^{13} \mathbf{C}$ NMR $\left(75 \mathrm{MHz}, \mathrm{CDCl}_{3}\right) \delta 136.3,128.5,128.2$, 127.8, 99.3, 66.7, 51.4, 46.5, 45.0, 38.7, 31.5, 28.9, 25.4; IR (neat, $\mathrm{cm}^{-1}$ ) 3374, 2934, 2857, 1603, 1327, 1142; MS (CI) m/z 343([M+H] $]^{+}$, base peak), 163; HRMS calcd for $\mathrm{C}_{16} \mathrm{H}_{27} \mathrm{~N}_{2} \mathrm{O}_{4} \mathrm{~S} 343.16914\left([\mathrm{M}+\mathrm{H}]^{+}\right)$, found 343.1685. 
LC analysis of the Mitsunobu product from rac-2-octanol.

Column; CHIRALCEL OB, $0.46 \mathrm{~cm} \phi \times 25 \mathrm{~cm} \mathrm{I.D.}$

Detector; UV-254nm

Eluent; $\mathrm{Hex} / \mathrm{i}-\operatorname{Pro}=9 / 1$

Flow rate; $0.5 \mathrm{ml} / \mathrm{min}$
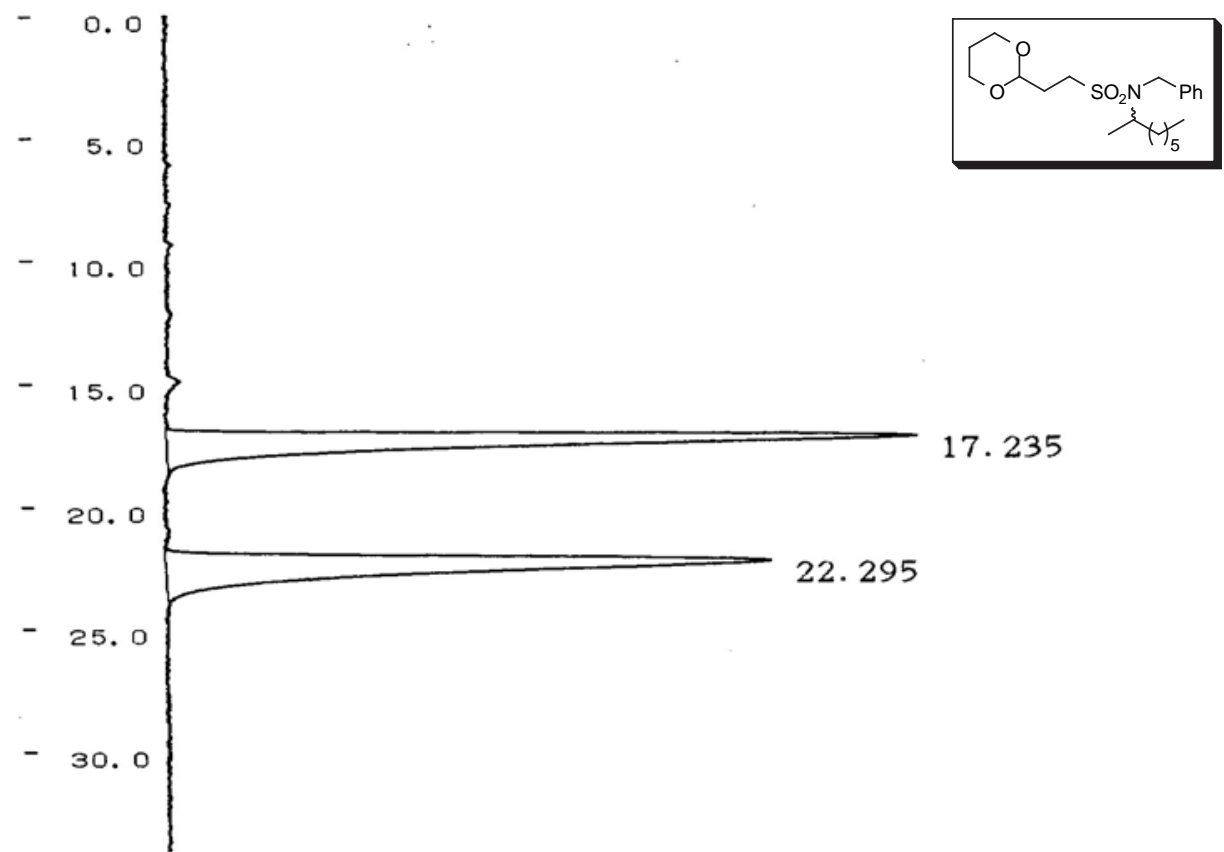

17. 235

\begin{tabular}{|c|c|c|c|c|c|}
\hline $\begin{array}{r}\text { PKNO } \\
1 \\
2\end{array}$ & $\begin{array}{c}\text { TIME } \\
17.235 \\
22.295\end{array}$ & $\begin{array}{l}\text { AREA } \\
1174819 \\
1174219\end{array}$ & $\begin{array}{l}\text { HE IGHT } \\
33091 \\
26634\end{array}$ & MK IDNO & $\begin{array}{l}\text { CONC } \\
50.0128 \\
49.9872\end{array}$ \\
\hline & TOTAL & 2349038 & 59725 & & 100 \\
\hline
\end{tabular}


LC analysis of the Mitsunobu product from (2S)-2-octanol.

Column; CHIRALCEL OD, $0.46 \mathrm{~cm} \phi \times 25 \mathrm{~cm} \mathrm{I.D.}$

Detector; UV $-254 \mathrm{~nm}$

Eluent; Hex $/ \mathrm{i}-\operatorname{Pro}=9 / 1$

Flow rate; $0.5 \mathrm{ml} / \mathrm{min}$

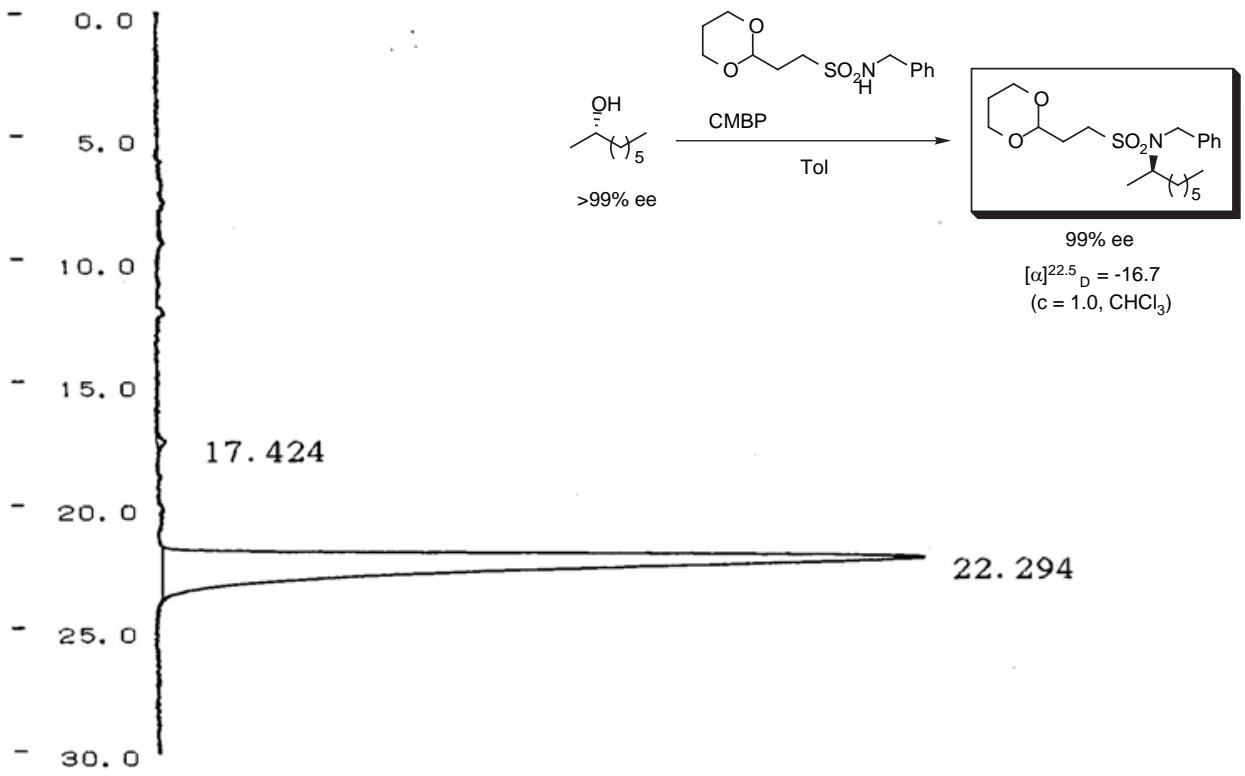

\begin{tabular}{|c|c|c|c|c|c|c|}
\hline $\begin{array}{r}\text { PKNO } \\
1 \\
2\end{array}$ & $\begin{array}{c}\text { TIME } \\
17.424 \\
22.294\end{array}$ & $\begin{array}{r}\text { AREA } \\
9268 \\
1512847\end{array}$ & $\begin{array}{r}\text { HE I GHT } \\
421 \\
33563\end{array}$ & MK & I DNO & $\begin{array}{r}\text { CONC } \\
0.6089 \\
99.3911\end{array}$ \\
\hline & TOTAL & 1522115 & 33984 & & & 100 \\
\hline
\end{tabular}


LC analysis of an authentic sample prepared via the traditional Mitsunobu reaction of $(2 S)$-2-octanol.

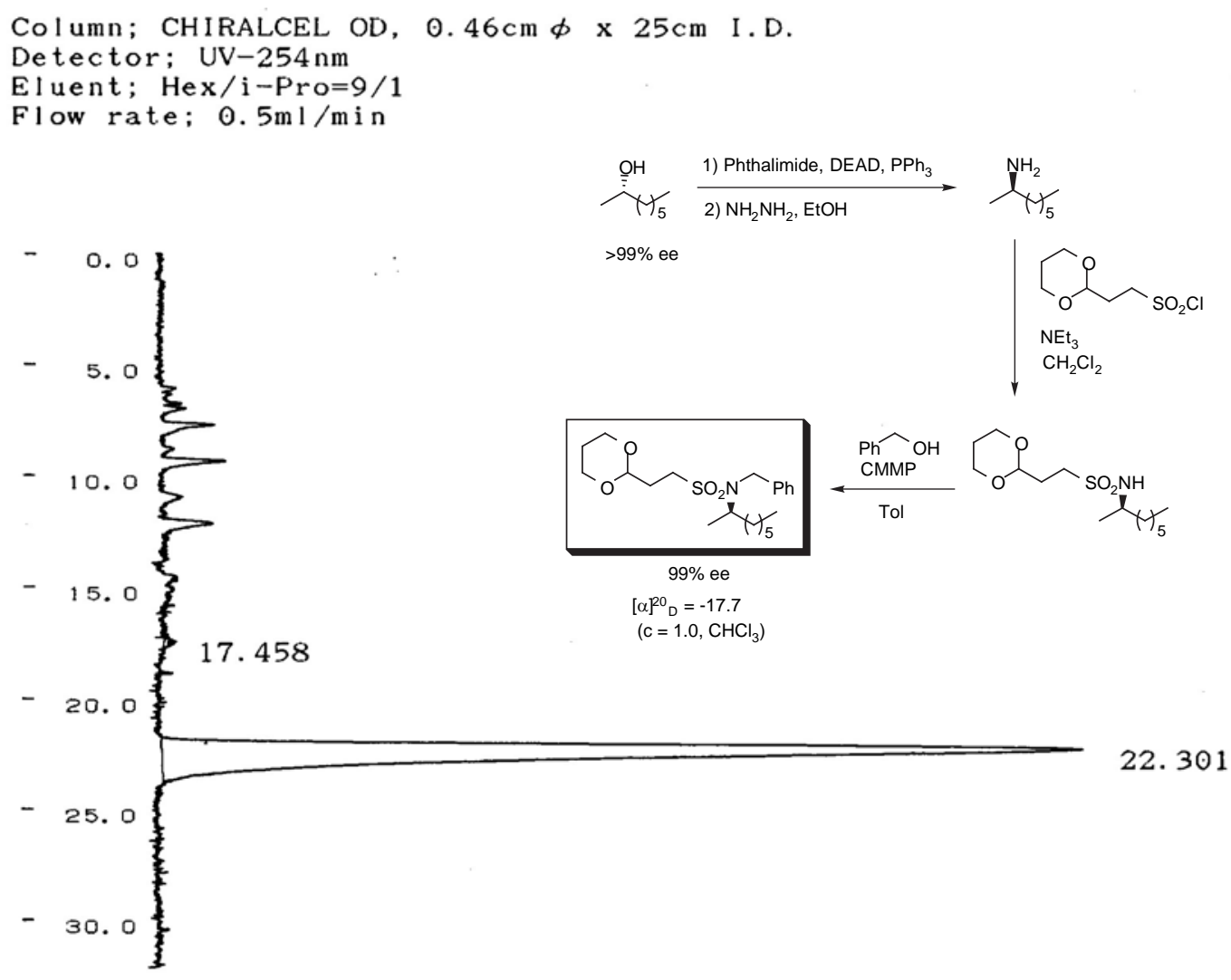

\begin{tabular}{rcrrrrr} 
PKNO & TIME & \multicolumn{1}{c}{ AREA } & HEIGHT & MK & IDNO & \multicolumn{2}{c}{ CONC } \\
1 & 17.458 & 4068 & 305 & & \\
2 & 22.301 & 966972 & 22380 & & 99.58189 \\
& TOTAL & 971040 & 22685 & & -100
\end{tabular}


$9 G-M-Y-04-1 H$

Pulse Sequence: s2pul

Solvent: $\mathrm{CDC} 13$

Ambient temperature

File: 9G-M-Y-04-1H092405 1833 INOVA-600 "NMR"

Relax. delay $1.000 \mathrm{sec}$

Pulse 38.7 degrees

Acq. time $4.000 \mathrm{sec}$

Width $4504.5 \mathrm{~Hz}$

244 repetitions

OBSERVE H1, $300.0445642 \mathrm{MHz}$

DATA PROCESSING

FT size 65536

Total time $1 \mathrm{hr}, 23 \mathrm{~min}, 23 \mathrm{sec}$
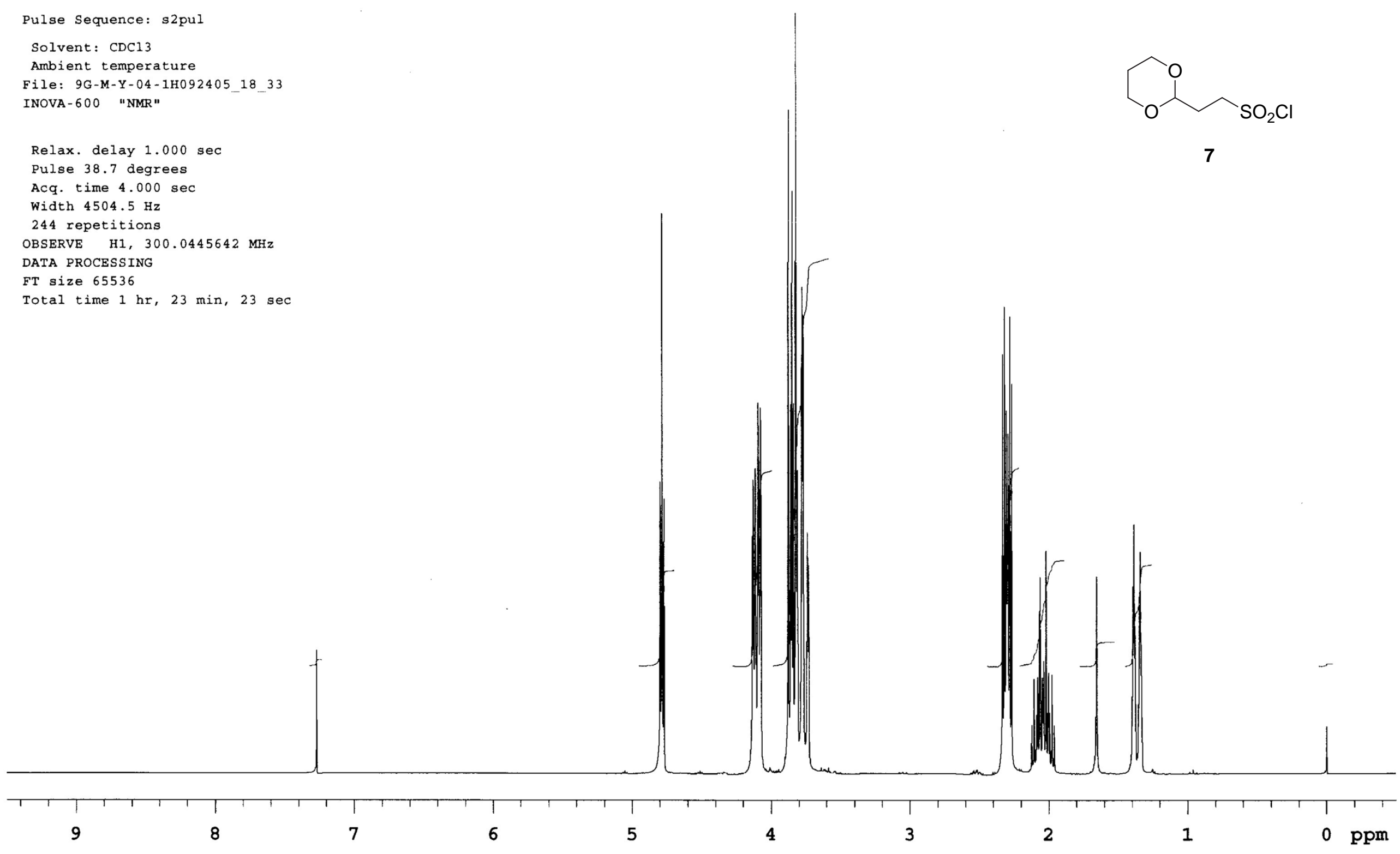
$9 G-M-Y-O I$

Pulse Sequence: 52pul

Solvent: $\operatorname{CDCl} 3$

Ambient temperature

File: 9 G-M-Y-02083105 1017

INOVA- 600 "NMR"

$\mathrm{SO}_{2} \mathrm{NH}_{2}$

Relax. delay 0.500 sec

Pulse 31.3 degrees

Acq. time $3.555 \mathrm{sec}$

Width $4500.5 \mathrm{~Hz}$

88 repetitions

OBSERV Hl, $300.0738738 \mathrm{MH}$

DATA PROCESSIM

FT size 32768

Total time $1 \mathrm{hr}, 7 \mathrm{~min}, 39 \mathrm{sec}$

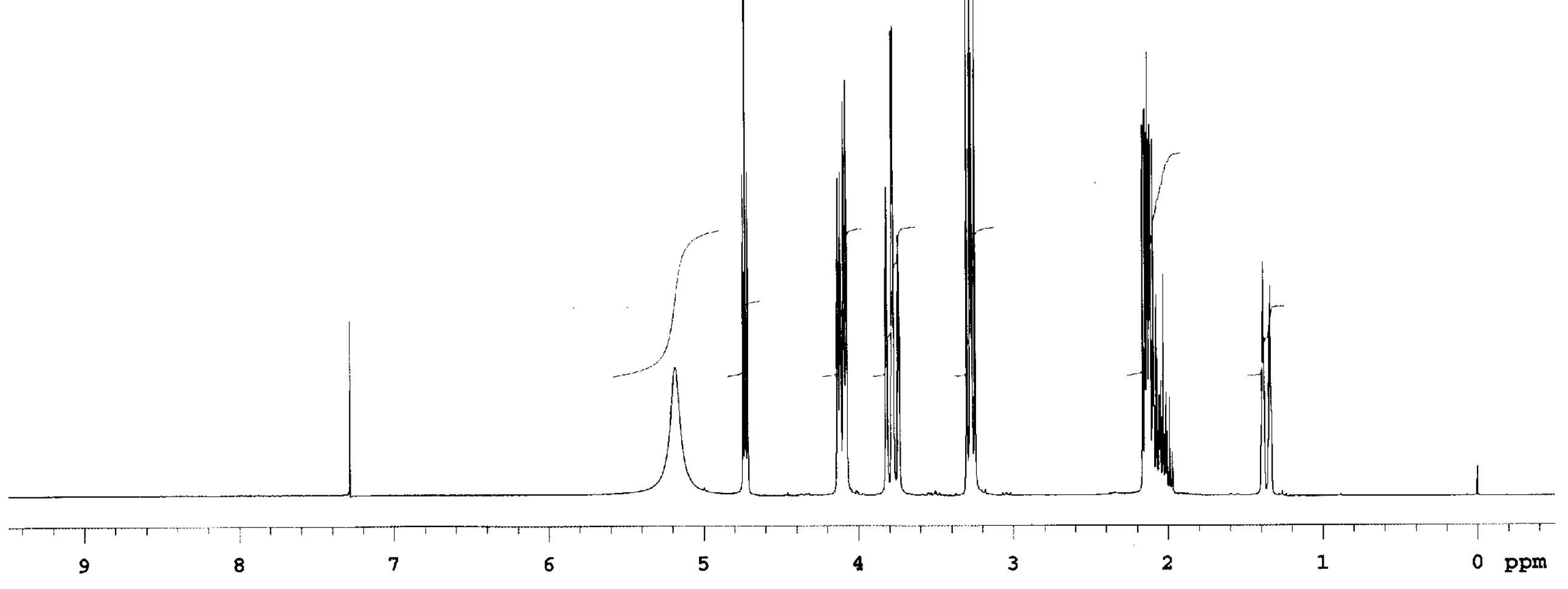




\section{$9 G-M-Y-05-1 H$}

Pulse Sequence: s2pul

Solvent: $\mathrm{CDCl} 3$

Ambient temperature

File: 9G-M-Y-05-1H092405 13 52 INOVA-600 "NMR"

Relax. delay $0.500 \mathrm{sec}$ Pulse 31.3 degrees

Acq. time $3.555 \mathrm{sec}$

width $4500.5 \mathrm{~Hz}$

96 repetitions

OBSERVE H1， $300.0738801 \mathrm{MHz}$

DATA PROCESSING

FT size 32768

Total time $1 \mathrm{hr}, 7 \mathrm{~min}, 39 \mathrm{sec}$

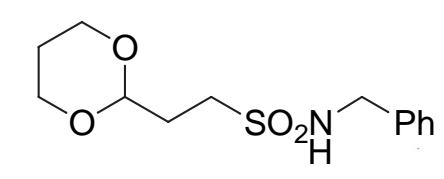

9a

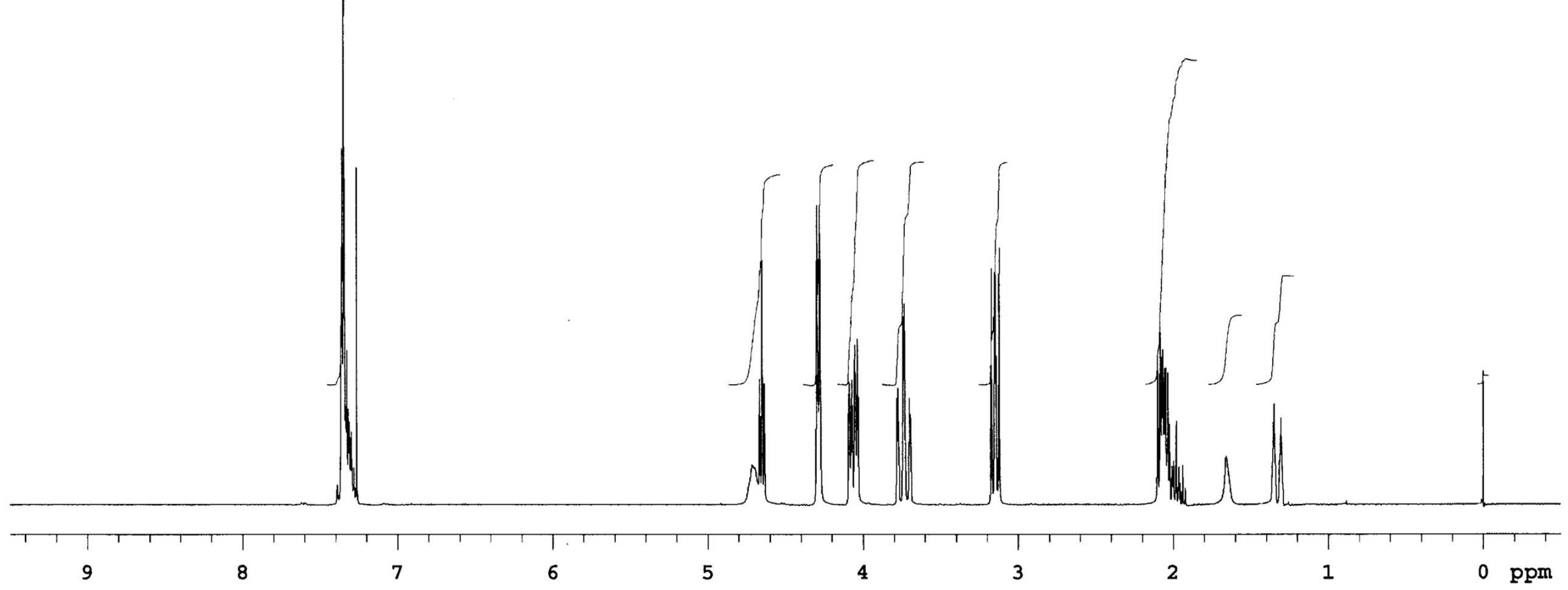


$9 G-3236-E-1 H$

Pulse Sequence: s2pul

Solvent: $\operatorname{CDCl} 3$

Ambient temperature

File: 9G-3236-E-1H081605 1611 INOVA- 600 "NMR"

Relax. delay $1.000 \mathrm{sec}$

Pulse 38.7 degrees

Acq. time $4.000 \mathrm{sec}$

Width $4504.5 \mathrm{~Hz}$

108 repetitions

OBSERVE H1，300.0445456 $\mathrm{MHz}$

DATA PROCESSING

FT size 65536

Total time $1 \mathrm{hr}, 23 \mathrm{~min}, 23 \mathrm{sec}$

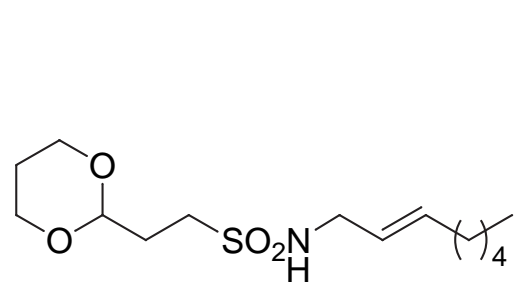

$9 b$

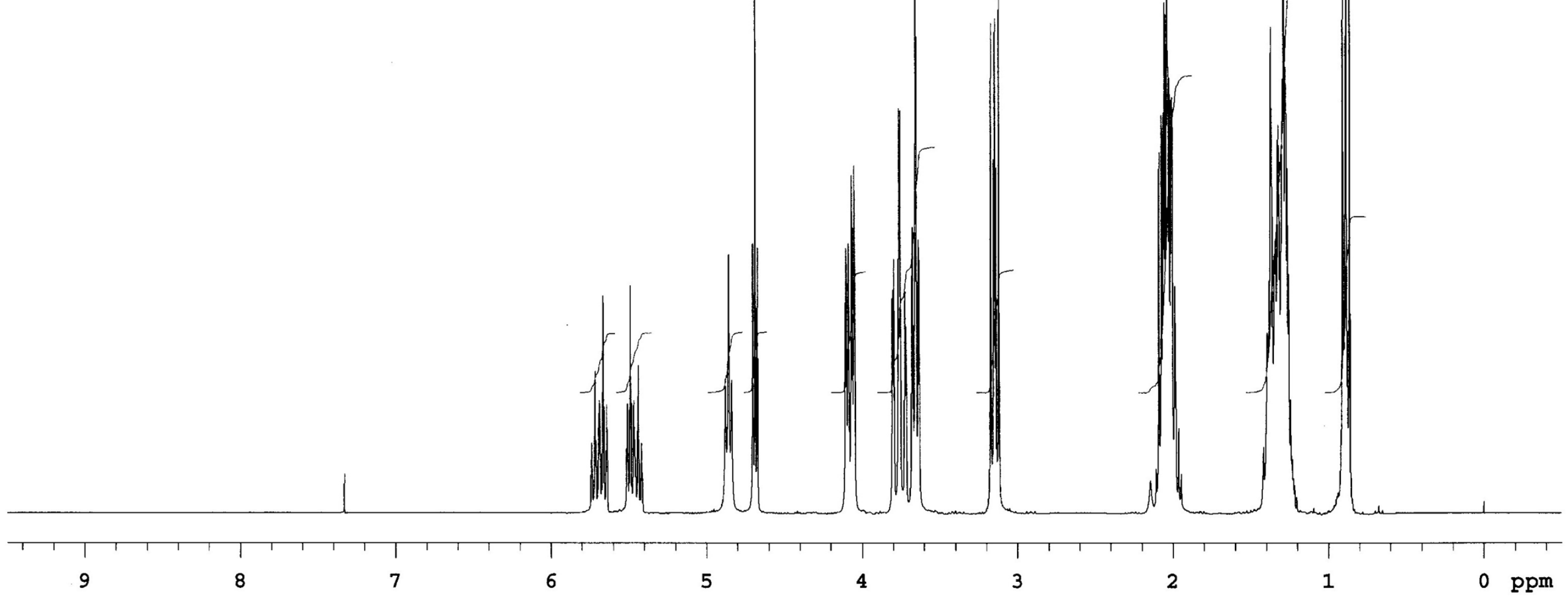


Pulse Sequence: s2pul

\section{Solvent: $\mathrm{CDCl}_{3}$}

Ambient temperature

File: 9G-M-Y-10-1H092405_16 10

INOVA-600 "NMR"

Relax. delay $1.000 \mathrm{sec}$

Pulse 38.7 degrees

Acq. time $4.000 \mathrm{sec}$

width $4504.5 \mathrm{~Hz}$

100 repetitions

OBSERVE HI, $300.0445651 \mathrm{MH}$

DATA PROCESSING

FT size 65536

Total time $1 \mathrm{hr}, 23 \mathrm{~min}, 23 \mathrm{sec}$

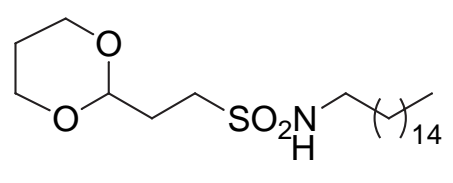

9c

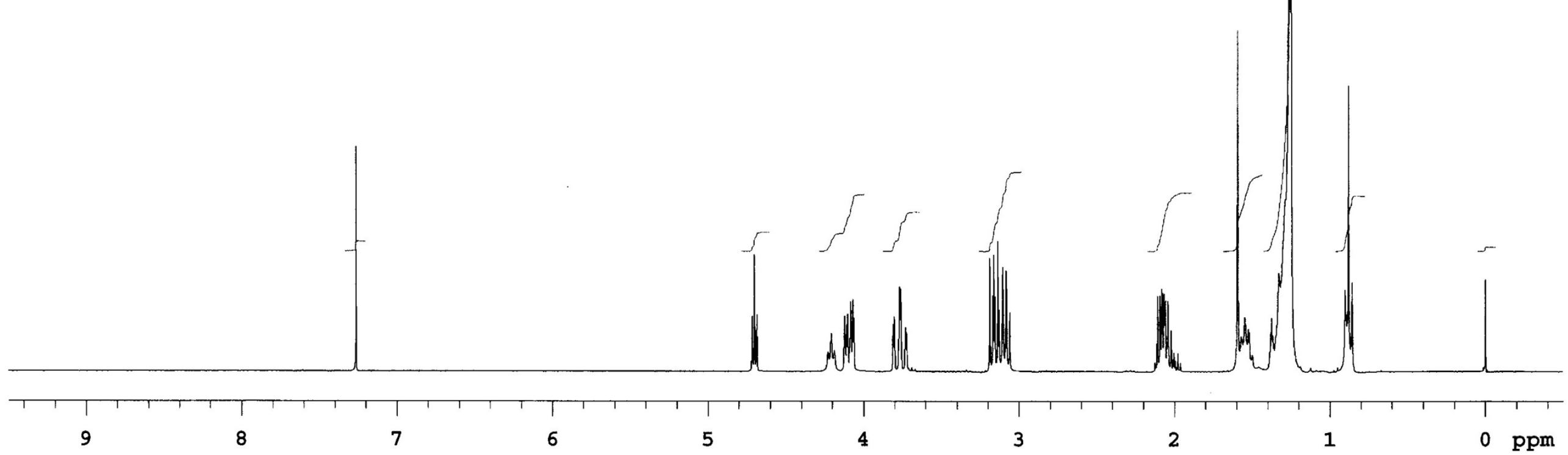


Pulse Sequence: s2pul

\section{Solvent: $\mathrm{CDCl} 3$}

Ambient temperature

File: 9G-M-Y-09-1H09240511_39

INOVA-600 "NMR"

Relax. delay $0.500 \mathrm{sec}$

Pulse 31.3 degrees

Acq. time $3.555 \mathrm{sec}$

Width $4500.5 \mathrm{~Hz}$

88 repetitions

OBSERVE H1, $300.0738779 \mathrm{MHz}$

DATA PROCESSING

FT size 32768

Total time $1 \mathrm{hr}, 7 \mathrm{~min}, 39 \mathrm{sec}$

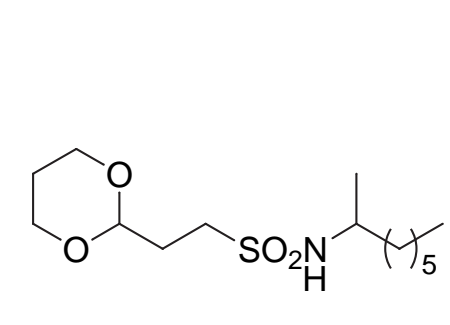

9d

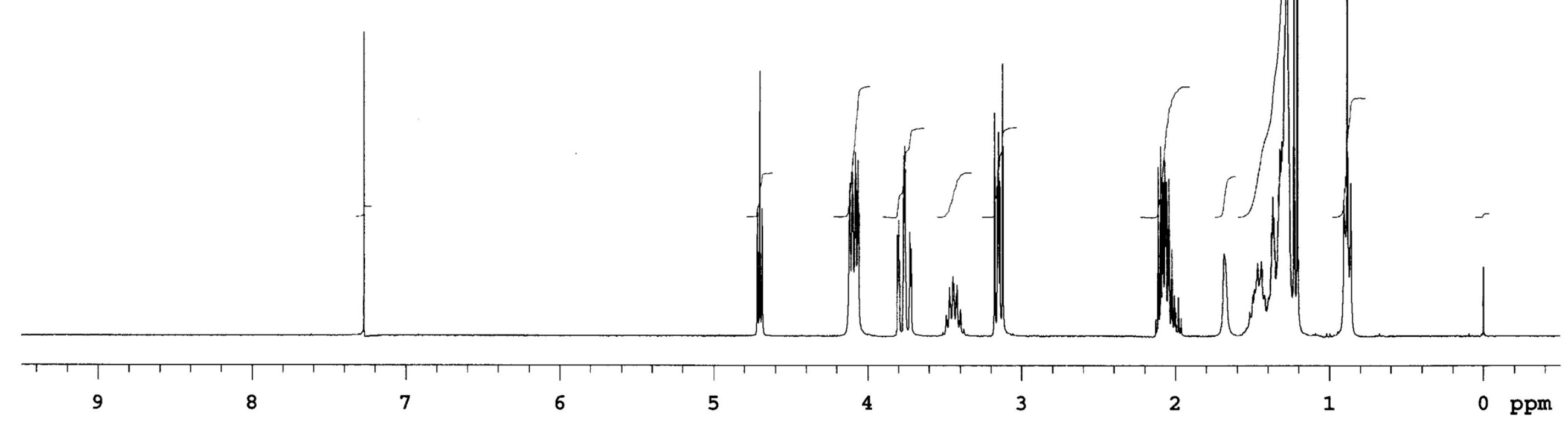


Pulse Sequence: $s 2 \mathrm{pul}$

Solvent: $\operatorname{CDCl} 3$

Ambient temperature

File: 9G-3025-B052505_13_41

INOVA-600 "NMR"

Relax. delay $1.445 \mathrm{sec}$

Pulse 78.3 degrees

Acq. time $3.555 \mathrm{sec}$

width $4500.5 \mathrm{~Hz}$

20 repetitions

OBSERVE H1, $300.0738801 \mathrm{MHz}$

DATA PROCESSING

FT size 32768

Total time $1 \mathrm{hr}, 23 \mathrm{~min}, 23 \mathrm{sec}$

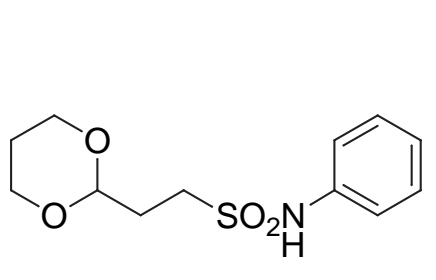

$9 e$

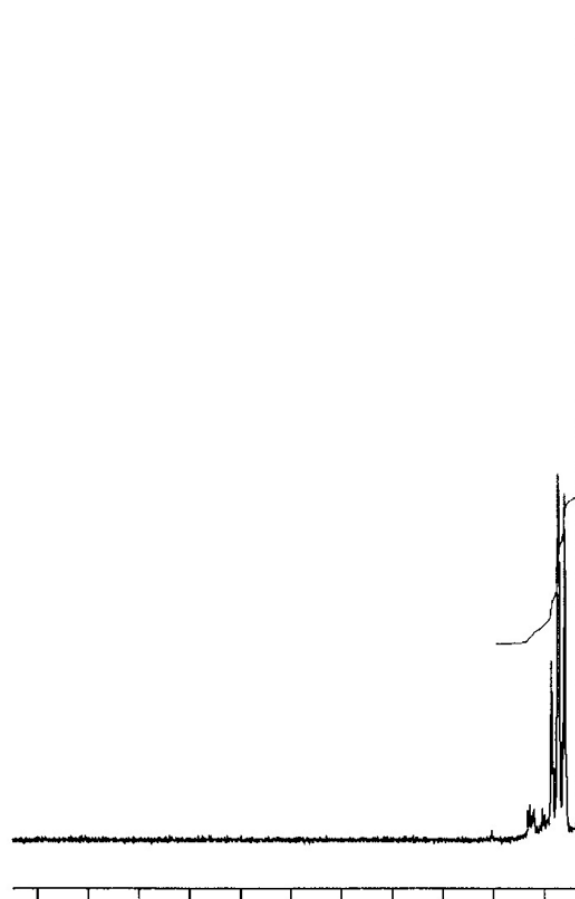


9G-3011-B 1H

Pr.1se Sequence: s2pul

Solvent: $\mathrm{CDCl} 3$

Ambient temperature

File: 9G-3011-1H100105 11 28

INOVA -600 "NMR"

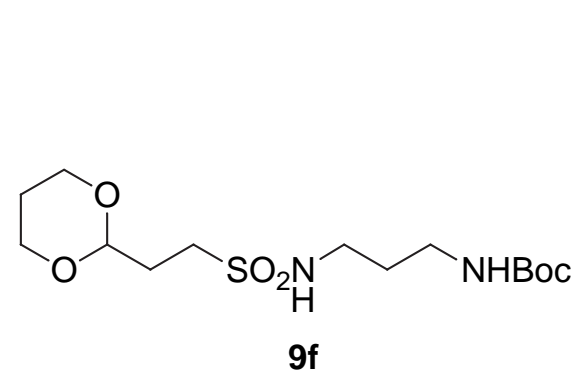

Relax. delay $1.000 \mathrm{sec}$

Pulse 38.7 degrees

Acq. time $4.000 \mathrm{sec}$

Width $4504.5 \mathrm{~Hz}$

112 repetitions

OBSERVE H1, $300.0445640 \mathrm{MHz}$

DATA PROCESSING

FT size 65536

Total time $1 \mathrm{hr}, 23 \mathrm{~min} ; 23 \mathrm{sec}$

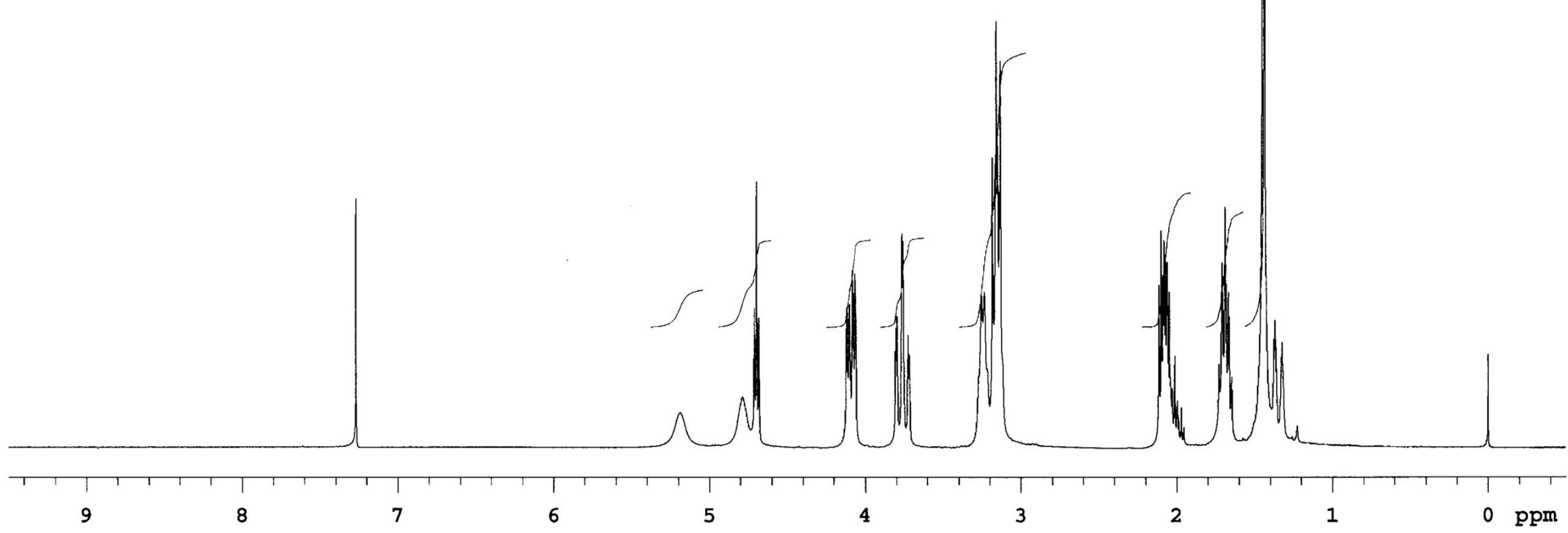


Pulse Sequence: s2pul

Solvent: $\mathrm{CDCl} 3$

Ambient temperature

File: 9G-M-Y-06-1H09240514 43

INOVA- 600 "NMR"

Relax. delay $0.500 \mathrm{sec}$

Pulse 31.3 degrees

Acq. time $3.555 \mathrm{sec}$

Width $4500.5 \mathrm{~Hz}$

92 repetitions

OBSERVE H1, $300.0738812 \mathrm{MHz}$

DATA PROCESSING

FT size 32768

Total time $1 \mathrm{hr}, 7 \mathrm{~min}, 39 \mathrm{sec}$

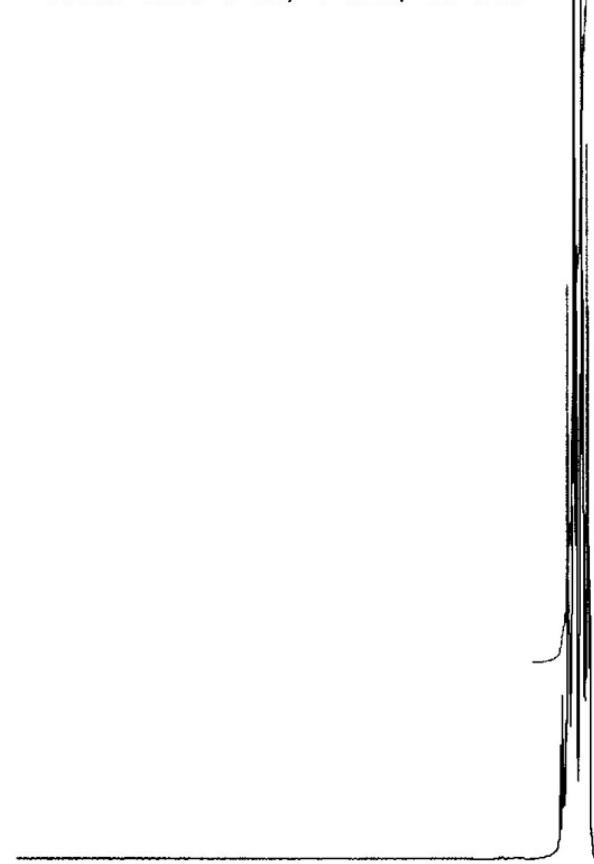

4

2

2

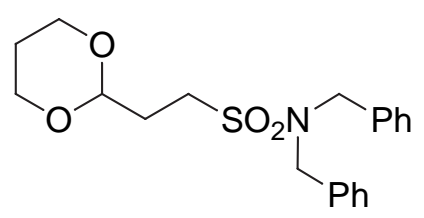

$10 a$ 
$9 \mathrm{G}-3236-\mathrm{C}-1 \mathrm{H}$

Pulse Sequence: s2pul

Solvent: $\mathrm{CDCl} 3$

Ambient temperature

File: 9G-3236-C-1H081605 1113

INOVA-600 "NMR"

Relax. delay $1.000 \mathrm{sec}$

Pulse 38.7 degrees

Acq. time $4.000 \mathrm{sec}$

Width $4504.5 \mathrm{~Hz}$

84 repetitions

OBSERVE H1, $300.0445620 \mathrm{MHz}$

DATA PROCESSING

FT size 65536

Total time $1 \mathrm{hr}, 23 \mathrm{~min}, 23 \mathrm{sec}$

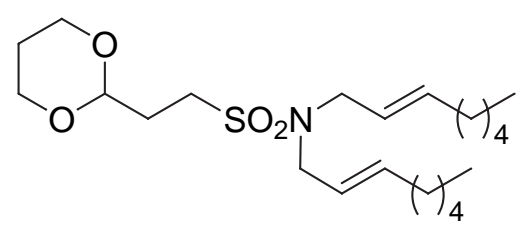

$10 b$

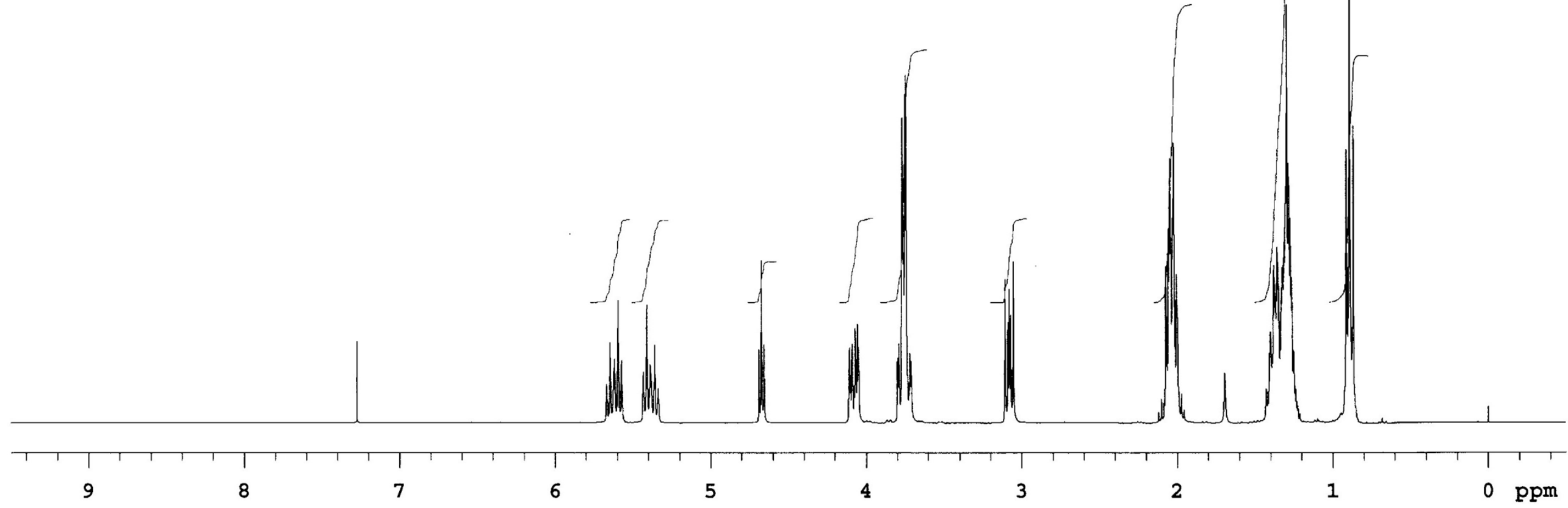


Fritse Sequence: 32pu:

Solvent: Coc13

Ambient temperature

File: 9G-M-Y-08-180529052141

INOVA-600 "NMR"

Relax. delay $0.500 \mathrm{sec}$

puise 31.3 degrees

Acq. time $3.555 \mathrm{sec}$

width $4500.5 \mathrm{~Hz}$

144 repetitions

OBSERVE H1, $300.0738796 \mathrm{MHz}$

DATA PROCESSING

FT size 32768

Total time $1 \mathrm{hr}, 7 \mathrm{~min}, 39 \mathrm{sec}$

( $\mathrm{hr}, 7 \mathrm{~min}, 39 \mathrm{sec}$
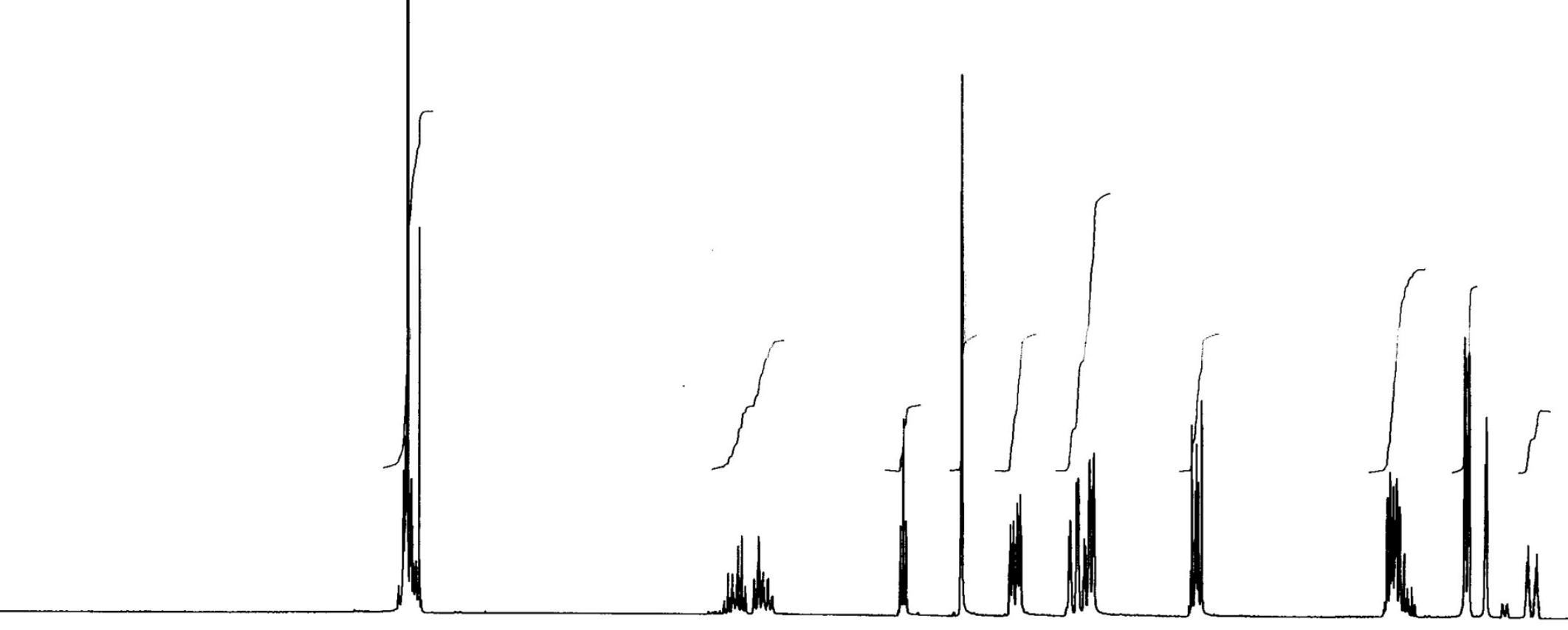

$10 c$

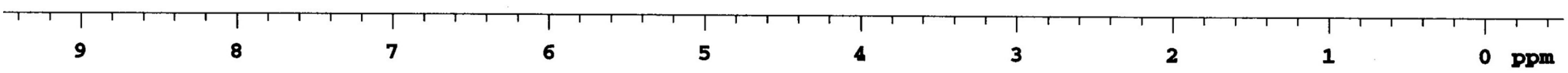


$9 G-M-Y-14-1 H$

Pulse Sequence: s2pul

Solvent: $\mathrm{CDCl} 3$

Ambient temperature

File: 9G-M-Y-14-1H092405 1659

File: 9 G-M-Y-14

Relax. delay $1.000 \mathrm{sec}$

Pulse 38.7 degrees

Acq. time $4.000 \mathrm{sec}$

width $4504.5 \mathrm{~Hz}$

144 repetitions

OBSERVE H1，300.0445658 MHz

DATA PROCESSING

FT size 65536

Total time $1 \mathrm{hr}, 23 \mathrm{~min}, 23 \mathrm{sec}$

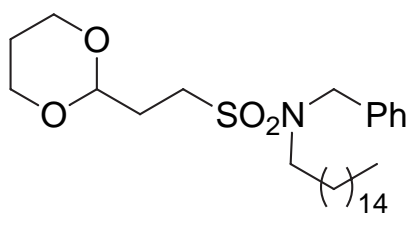

$10 d$

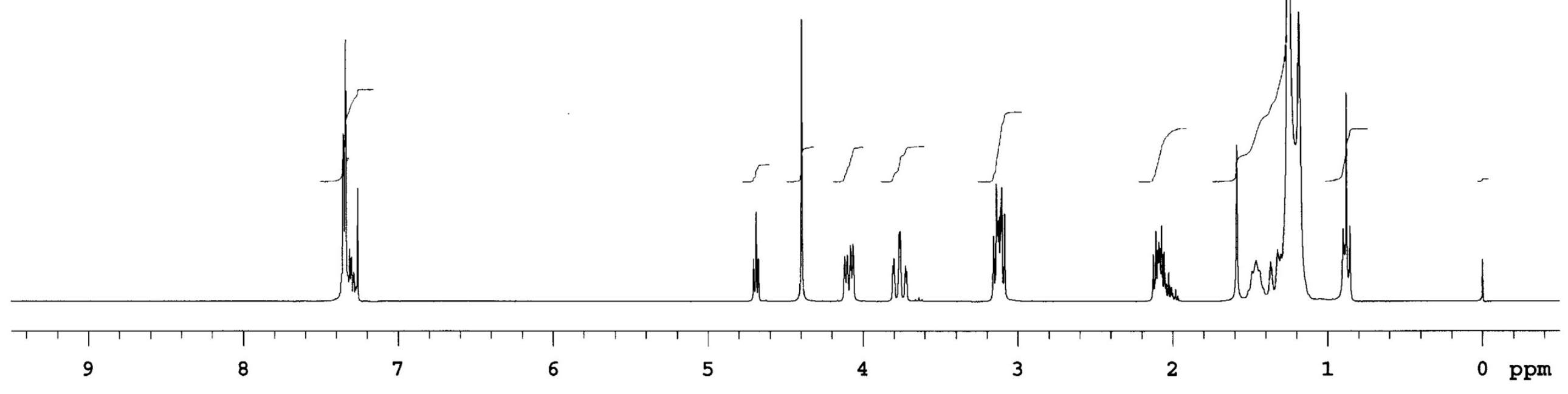


STANDARD IH OBSERVE

Pulse Sequence: s2pul

Solvent: $\operatorname{CDCl} 3$

Ambient temperature

File: 9G-M-Y-24-1H083105 1153

INOVA-600 "NMR"

Relax. delay $1.000 \mathrm{sec}$

Pulse 38.7 degrees

Acq. time $4.000 \mathrm{sec}$

Width $4504.5 \mathrm{~Hz}$

OBSERVE H1，300.0445603 MHz

DATA PROCESSING

FT size 65536

Total time $1 \mathrm{hr}, 23 \mathrm{~min}, 23 \mathrm{sec}$

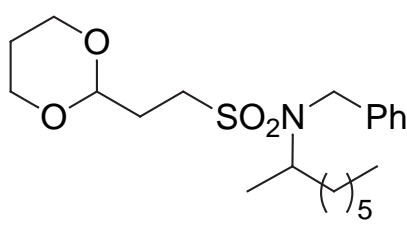

$10 e$

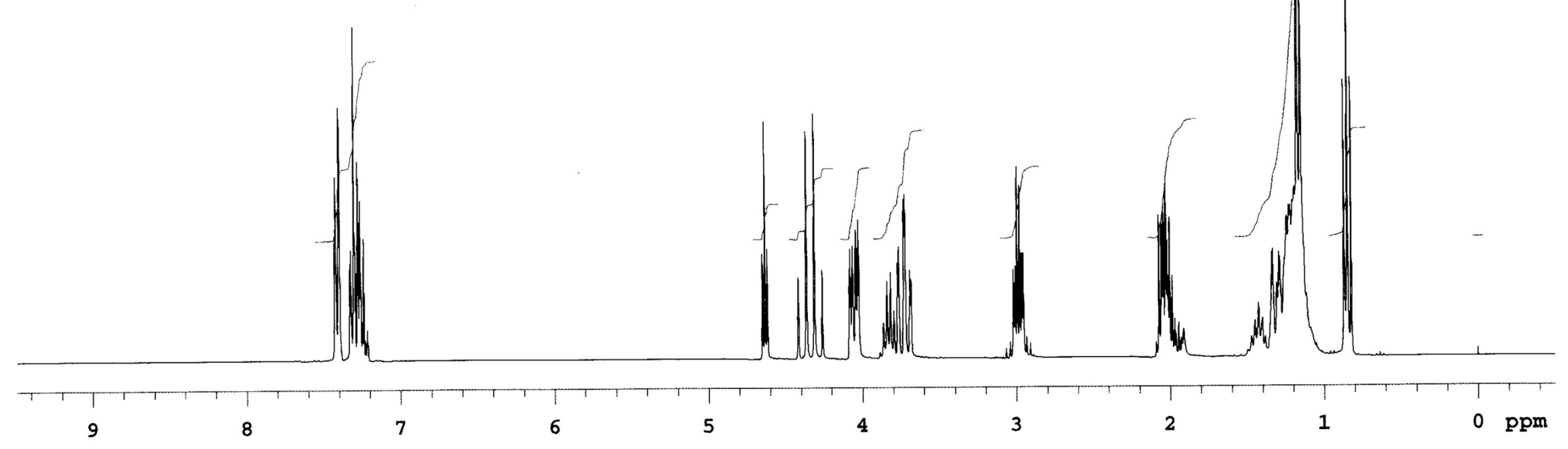


$9 G-M-Y-25$

Pulse Sequence: s2pul

Solvent: $\mathrm{CDC} 33$

Ambient temperature

File: 9G-M-Y-25-1H090105 0039

INOVA-600 "NMR"

Relax. delay $1.000 \mathrm{sec}$

Pulse 38.7 degrees

Acq. time $4.000 \mathrm{sec}$

Width $4504.5 \mathrm{~Hz}$

88 repetitions

OBSERVE HI $300.0445579 \mathrm{MHz}$

DATA PROCESSING

FT size 65536

Total time $1 \mathrm{hr}, 23 \mathrm{~min}, 23 \mathrm{sec}$

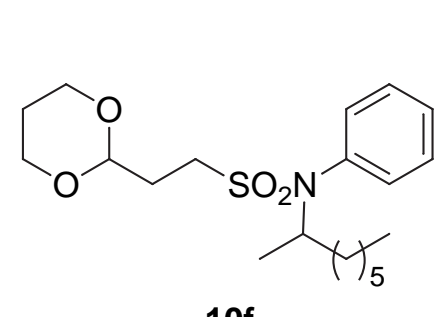

$10 f$
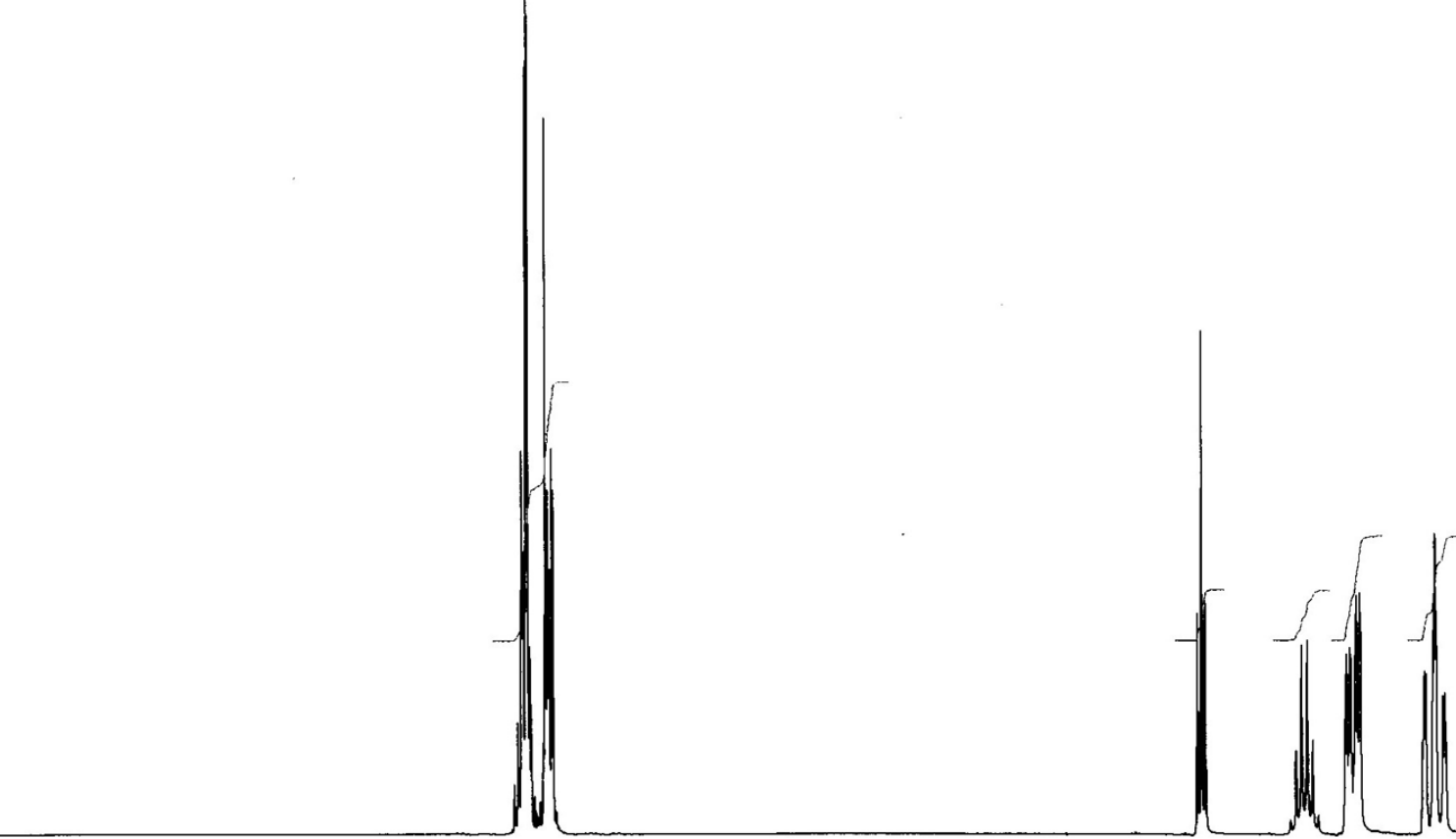

7

6

4

ppm 
Pulse Sequence: s2pul

Solvent: $\mathrm{CDC13}$

Ambient temperature

File: 9G-3125-1H092405 1203

INOVA-600 "NMR"

Relax. delay $0.500 \mathrm{sec}$

Pulse 31.3 degrees

Acq. time $3.555 \mathrm{sec}$

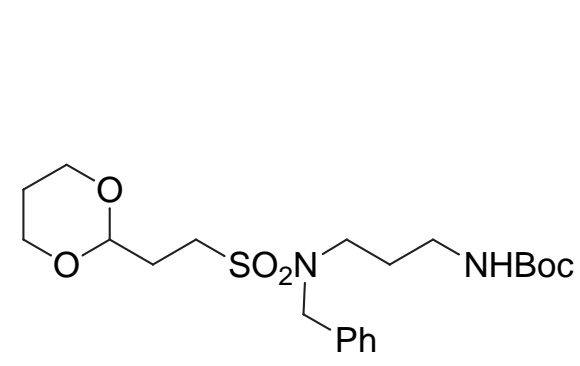

Width $4500.5 \mathrm{~Hz}$

$10 \mathrm{~g}$

tions

OBSERVE H1, $300.0738771 \mathrm{MHz}$

DATA PROCESSING

FT size 32768

Total time $1 \mathrm{hr}, 7 \mathrm{~min}, 39 \mathrm{sec}$

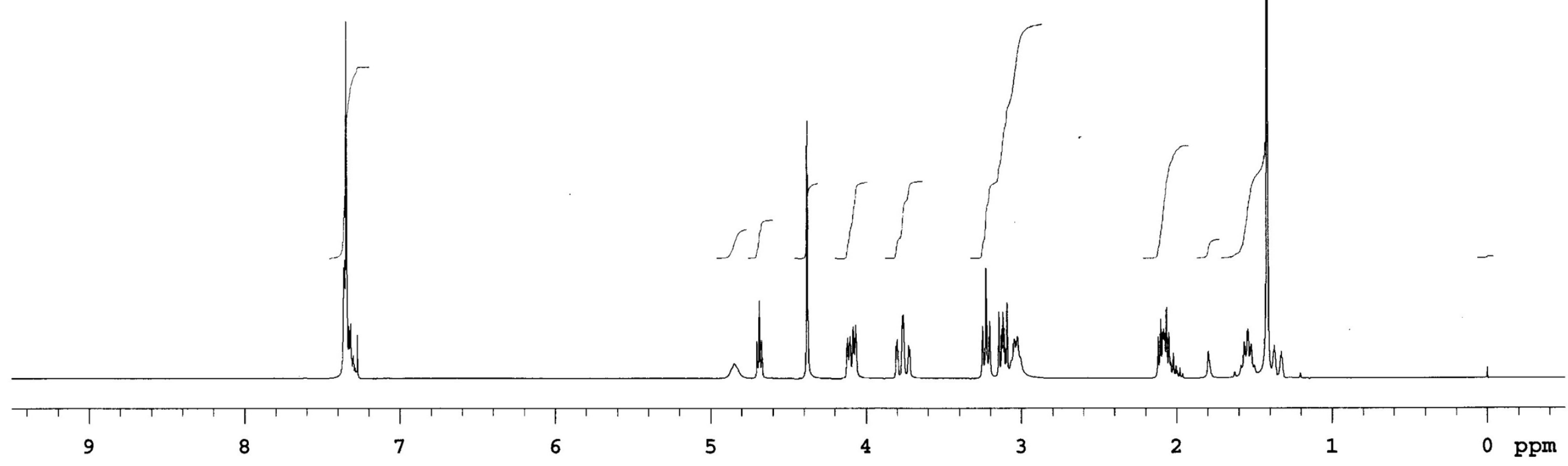


$9 \mathrm{G}-3320-\mathrm{B}$

Pulse Sequence: s2pul

Solvent: $\mathrm{CDCl} 3$

Ambient temperature

File: 9G-3320-B091905 2218

INOVA-600 "NMR"

Relax. delay $0.500 \mathrm{sec}$

Pulse 31.3 degrees

Acq. time $3.555 \mathrm{sec}$

width $4500.5 \mathrm{~Hz}$

32 repetitions

OBSERVE H1, $300.0738702 \mathrm{MHz}$

DATA PROCESSING

FT size 32768

Total time $1 \mathrm{hr}, 7 \mathrm{~min}, 39 \mathrm{sec}$

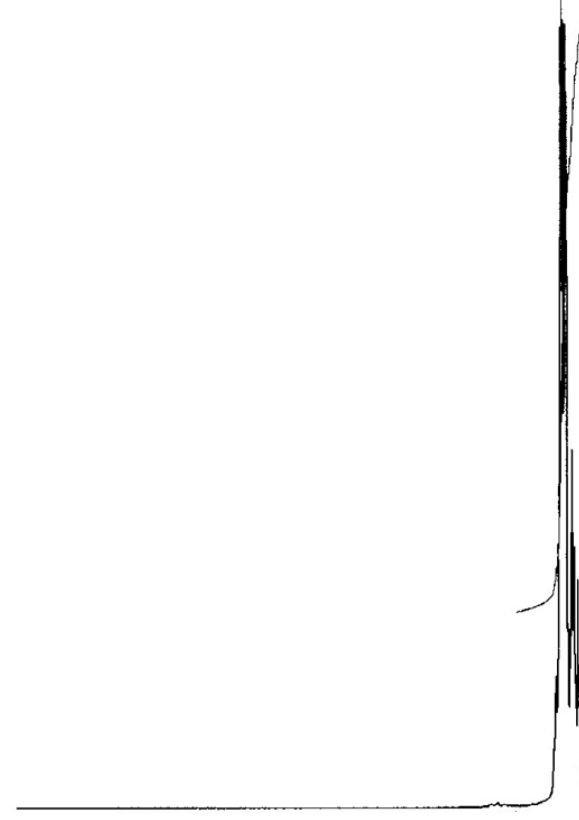

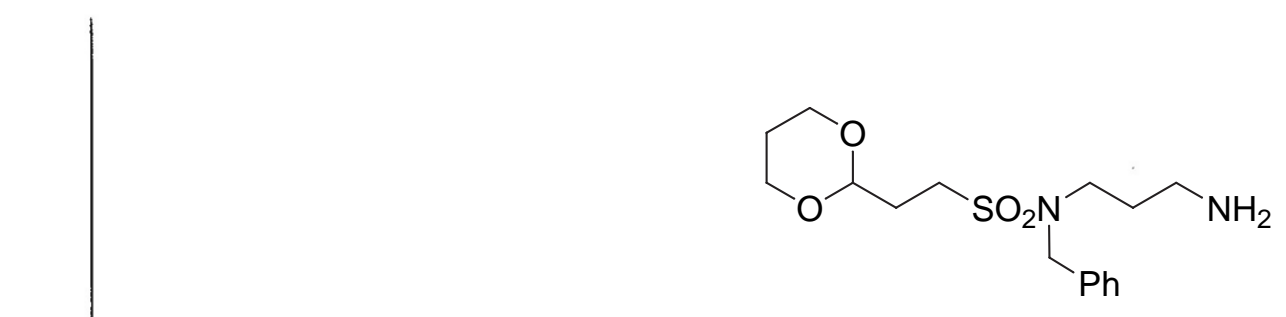

11

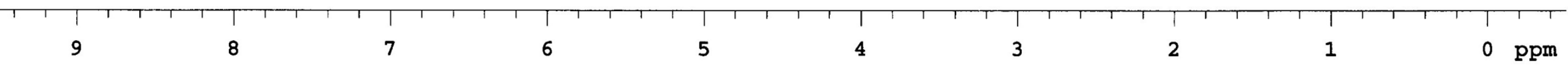

